Luciana Bivanco de Lima Lorentz

\title{
Estresse laboral e aterosclerose subclínica: análise transversal do Estudo Longitudinal de Saúde (ELSA- Brasil)
}

Tese apresentada à Faculdade de Medicina

da Universidade de São Paulo

para obtenção do título de Doutor em Ciências

Programa de Ciências Médicas

Orientadora: Profa. Dra. Isabela Judith Martins Benseñor

São Paulo

2019 


\section{Luciana Bivanco de Lima Lorentz}

\section{Estresse laboral e aterosclerose subclínica: análise transversal do Estudo Longitudinal de Saúde (ELSA- Brasil)}

Tese apresentada à Faculdade de Medicina

da Universidade de São Paulo

para obtenção do título de Doutor em Ciências

Programa de Ciências Médicas

Orientadora: Profa. Dra. Isabela Judith Martins

Benseñor

São Paulo

2019 
Dados Internacionais de Catalogação na Publicação (CIP)

Preparada pela Biblioteca da

Faculdade de Medicina da Universidade de São Paulo

Creprodução autorizada pelo autor

Lorentz, Luciana Bivanco de Lima

Estresse laboral e aterosclerose subclínica :

análise transversal do Estudo Longitudinal de Saúde

(ELSA-Brasil) / Luciana Bivanco de Lima Lorentz. --

São Paulo, 2019.

Tese(doutorado)--Faculdade de Medicina da

Universidade de São Paulo.

Programa de Ciências Médicas. Área de

Concentração: Educação e Saúde.

Orientadora: Isabela Judith Martins Benseñor.

Descritores: 1.Estresse ocupacional

2.Aterosclerose 3.Fatores de risco 4.Aterosclerose

subclínica 5.Calcificação de artéria coronariana

6.Espessura intima-média carotídea 7.Doenças

cardiovasculares

$\mathrm{USP} / \mathrm{FM} / \mathrm{DBD}-453 / 19$

Responsável: Erinalva da Conceição Batista, CRB-8 6755 



\section{AGRADECIMENTOS}

Agradeço imensamente à Dra. Isabela, por ter me acompanhado e guiado durante toda a minha formação médica, como minha primeira professora de Clínica Médica, primeira tutora, e hoje orientadora. A Dra. é uma inspiração!

Agradeço ao Dr. César Kubiak, chefe e querido colega, por todo apoio e tolerância durante esse percurso.

Muito obrigada à toda minha família, mas em especial ao meu pai João, que me ensinou tudo o que realmente importa nessa vida, e à minha irmã Danielle, mulher batalhadora e inteligente em quem me espelho e sem a qual esse doutorado nunca teria saído.

Agradeço à minha amiga-irmã-vizinha Ana Paula, pelo companheirismo, cafés com bolo, pelos sopros de motivação e ombro amigo de sempre. Ao Gustavo, pelo apoio com a Maria e por dividir comigo tão lealmente essa tarefa fantástica que é criar uma filha. Muito obrigada!

E por último, mas não menos importante, agradeço à minha filha Maria Flor, pelo eterno aprendizado e por trazer significado a tudo isso. 


\section{SUMÁRIO}

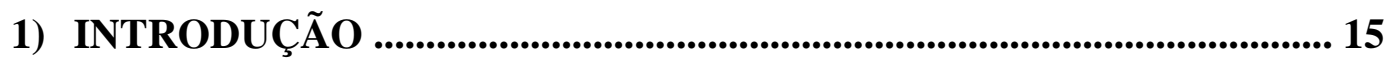

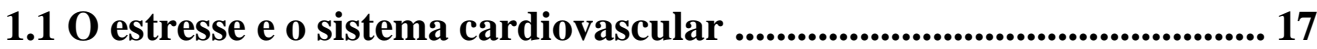

1.2 Estresse relacionado ao trabalho .................................................. 18

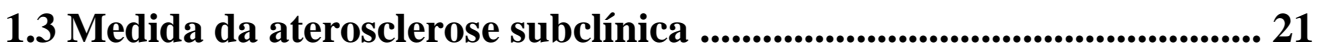

1.4 Espessura Íntima-Média de Carótidas (EIMC) ............................... 23

1.5 Tomografia Computadorizada com Mensuração de Cálcio

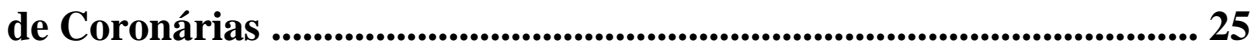

1.6 Estresse no trabalho e aterosclerose subclínica .................................. 26

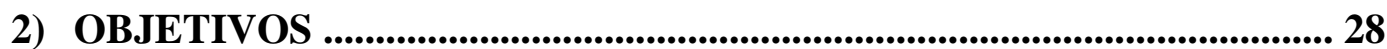

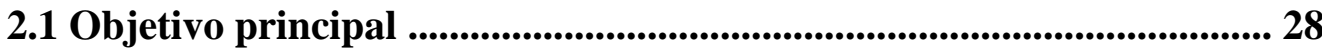

2.2 Objetivos secundários .............................................................................. 28

2.3 Hipótese do estudo ................................................................................... 28

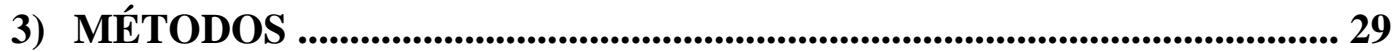

3.1 Estresse no trabalho ............................................................................. 30

3.2 Mensuração da aterosclerose subclínica ............................................... 33

3.2.1 Escore de cálcio ........................................................................... 33

3.2.2 Espessura íntima-média de carótidas ..................................... 34

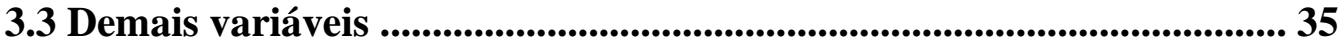




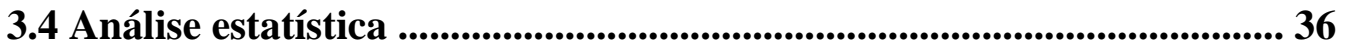

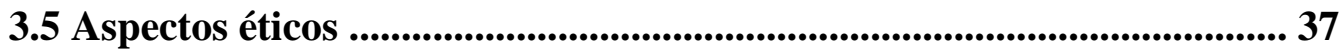

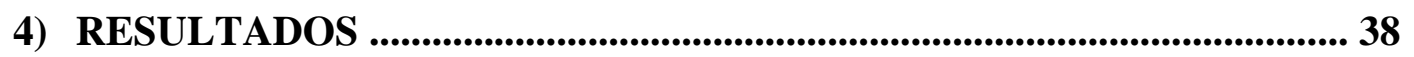

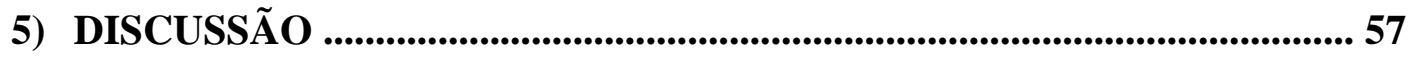

5.1 Estresse no trabalho e espessura íntima-média de carótidas ................ 57

5.2 Estresse no trabalho e escore de cálcio em artérias coronárias ............ 64

5.3 Limitações do estudo ..................................................................................66

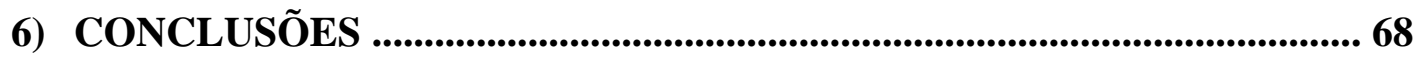

7) REFER̂̂NCIAS BIBLIOGRÁFICAS ....................................................... 69

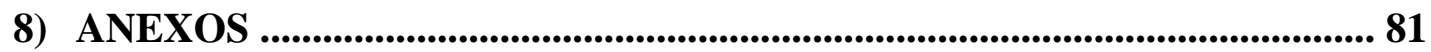




\section{LISTA DE ABREVIATURAS}

CAC - Calcificação de artéria coronária

DC - Demanda-controle

DCS - Escala Sueca de Demanda-Controle-Apoio Social

DER - Desequilíbrio esforço-recompensa

DP - Desvio-padrão

ECG - Eletrocardiograma

EIMC - Espessura Íntima-Média de Carótidas

ELSA - Estudo Longitudinal de Saúde do Adulto

FR - Fatores de risco

IC $95 \%$ - Intervalos de confiança $95 \%$

IMC - Índice de massa corporal

IPAQ - International Physical Activity Questionnaire

JCQ - Job Content Questionnaire

MIA - Medical Imaging Applications

RC - Razão de chances

SDSQ - Swedish Demand-Control-Support Questionnaire

UI - Unidades 


\section{LISTA DE FIGURAS}

Figura 1 - Esquema do Modelo de Demanda- Controle de Karasek ..................... 31

\section{LISTA DE QUADROS}

Quadro 1 - Amplitude e classificação dos domínios do estresse no trabalho 33 


\section{LISTA DE TABELAS}

Tabela 1. Características sociodemográficas e perfil de fatores de risco em participantes do estudo ELSA-Brasil de acordo com o sexo. Pág.39

Tabela 2. Nível de aterosclerose subclínica e dos domínios de estresse no trabalho em participantes do estudo ELSA-Brasil de acordo com o sexo. Pág.41

Tabela 3. Características gerais dos participantes do estudo ELSA-Brasil de acordo com as categorias de estresse no trabalho. Pág.43

Tabela 4. Modelos de regressão logística com Razões de Chance (RC) com Intervalos de Confiança a 95\% (IC 95\%) com CAC $>0$ e $\geq 100$ de acordo com os tercis de demanda, controle e suporte social. Pág.45

Tabela 5. Modelos de regressão logística com Razões de Chance (RC) com Intervalos de Confiança a 95\% (IC 95\%) com a EIMC (média dos valores médios) >P75\% e a EIMC (média dos valores máximos) de acordo com os tercis de demanda, controle e suporte social. Pág.46

Tabela 6. Modelos de regressão logística com Razões de Chance (RC) com Intervalos de Confiança a 95\% (IC 95\%) com CAC > 0 e $\geq 100$ de acordo com tercis de demanda, habilidade, autoridade decisória e suporte social. Pág.47

Tabela 7. Modelos de regressão logística com Razões de Chance (RC) com Intervalos de Confiança a 95\% (IC 95\%) com a EIMC (média dos valores médios) >P75th e a EIMC (média dos valores máximos) de acordo com os tercis de demanda, habilidades, autoridade decisória e suporte social. Pág.49

Tabela 8. Beta (Intervalo de Confiança a 95\%) da associação entre tercis de demanda, controle e apoio social e espessura de íntima-média de carótidas (média dos valores médios) sem ajuste, ajustado por fatores sociodemográficos e após ajuste multivariado. Pág.51

Tabela 9. Beta e Intervalo de Confiança a $95 \%$ da associação de tercis de demanda, uso de habilidade, autoridade decisória e apoio social com a espessura de íntima-média de carótidas (média dos valores médios) sem ajuste, ajustado por fatores sociodemográficos e ajuste multivariado. Pág.52

Tabela 10. Beta (Intervalo de Confiança a 95\%) da associação entre tercis de demanda, controle, apoio social e Espessura de íntima-média de carótidas (média dos valores máximos) sem ajuste, ajustado por fatores sociodemográficos e após ajuste multivariado. Pág.54

Tabela 11. Beta e Intervalo de Confiança a 95\% da associação de tercis de demanda, uso de habilidade, autoridade decisória e apoio social com a espessura de íntima-média de carótidas (média dos valores máximos) sem ajuste, ajustado por fatores sociodemográficos e ajuste multivariado. Pág.55 


\section{RESUMO}

Lorentz LBL. Estresse laboral e aterosclerose subclínica: análise transversal do Estudo Longitudinal de Saúde (ELSA-Brasil) [tese]. São Paulo: Faculdade de Medicina, Universidade de São Paulo; 2019.

Introdução: Poucos estudos avaliaram a associação entre estresse no trabalho com aterosclerose subclínica. O objetivo desta análise é estudar esta associação no Estudo Longitudinal de Saúde do Adulto (ELSA-Brasil). Métodos: Modelos de regressão logística foram construídos considerando-se o estresse no trabalho como as variáveis independentes e o escore de cálcio $(\mathrm{CAC}>0$ e $\mathrm{CAC} \geq 100)$ e a espessura íntima-média de carótidas (EIMC) avaliada como variável categórica (EIMC >P75\%) e como variável contínua como as variáveis dependentes. Para demanda, a referência foi a categoria baixa enquanto para uso de habilidades, autoridade decisória e suporte social a referência foi a categoria alta. As Razões de Chance (RC) com os respectivos Intervalos de Confiança a 95\% (IC 95\%) foram apresentados sem ajuste, ajustados por idade, sexo, raça e nível educacional (variáveis sociodemográficas), com posterior ajuste para índice de Massa Corpórea (IMC), hipertensão, diabetes, dislipidemia, tabagismo, uso de álcool e atividade física. Modelos de regressão linear foram construídos usando os mesmos ajustes. Resultados: Após ajuste multivariado, a RC para CAC >0 para alta demanda foi 1,17 (IC 95\%, 0,90-1,51); para uso de habilidades foi 0.99 (IC 95\%, 0,63-1.30); para autoridade decisória foi 1,08 (IC 95\%, 0,83-1,40); e baixo suporte social foi 0,91 (IC 95\%, 0,751,25). Resultados similares foram obtidos para o CAC $\geq 100$. Para a EIMC, após o mesmo ajuste multivariado e usando as mesmas referências, a RC para alta demanda foi de 1,06 (IC 95\%CI, 0,83-1,36); para uso de habilidades foi 1,05 (IC 95\%, 0,81-1,36); para autoridade decisória foi 0,89 (IC 95\%, 0,69-1,14); e para suporte social baixo foi 1,12 (IC 
95\%, 0,88-1,43). Usando modelos lineares, houve uma associação entre autoridade decisória baixa e média e com suporte social baixo e médio com níveis mais baixos de EIMC, mas as associações perderam significância após ajuste pelas variáveis sociodemográficas. Conclusão: Os resultados indicam uma ausência de associação entre estresse no trabalho e aterosclerose subclínica no ELSA-Brasil.

Descritores: Estresse ocupacional; Aterosclerose; Fatores de risco; Aterosclerose subclínica; Calcificação de artéria coronariana; Espessura intima-média carotídea, Doenças cardiovasculares. 


\begin{abstract}
Lorentz LBL. Laboral stress and subclinical atherosclerosis: transversal analysis of Estudo Longitudinal de Saúde (ELSA-Brazil) [thesis]. São Paulo: "Faculdade de Medicina, Universidade de São Paulo"; 2019.
\end{abstract}

Background: Few studies evaluated the relationship of job stress with subclinical atherosclerosis in ELSA-Brazil. We aimed to evaluate the association of job stress and subclinical atherosclerosis. Methods: Logistic regression models were built considering job stress domains as independent variables and CAC score $(>0 ; \geq 100)$ and CIMT $\left(>\mathrm{P} 75^{\text {th }}\right.$ or as a continuous variable) as the dependent ones. For demand the reference was low category while for skill discretion, decision authority and social support was high category. Odds ratio (OR) and 95\% Confidence Interval (95\% CI) were presented crude, adjusted for sage, sex, race and education, with further multivariate adjustment for BMI, hypertension, diabetes, dyslipidemia, smoking, alcohol intake, and physical activity. Linear regression models were built using the same adjustments. Results: After multivariate adjustment, the OR of CAC >0 for high demand was 1.17 (0.90-1.51); for low skill discretion was 0.99 (95\%CI, 0.63-1.30); for decision authority was 1.08 (95\%CI, 0.83-1.40); and for low social support was 0.91 (95\%CI, 0.75-1.25). Similar results were obtained for $\mathrm{CAC} \geq 100$. For $\mathrm{CIMT}$, after multivariate adjustment using the same references, the OR for high demand was 1.06 (95\%CI, 0.83-1.36); low skill discretion was 1.05 (95\%CI, 0.81-1.36); for low decision authority was 0.89 (95\%CI, 0.691.14); and for low social support was 1.12 (95\%CI, 0.88-1.43). Using linear regression models, there was an association of low and middle decision authority and social support with lower CIMT levels, but all results lost significance after further adjustment for 
sociodemographic variables. Conclusion: Our results did not support an association of job stress with subclinical atherosclerosis.

Descriptors: Occupational stress; Atherosclerosis; Risk factors; Subclinical atherosclerosis; Coronary artery calcification; Carotid intima-media thickness; Cardiovascular diseases. 


\section{Introdução}

As doenças cardiovasculares são a principal causa de mortalidade no mundo, sendo responsáveis por 17,6 milhões de óbitos em 2016 (Global Burden of Diseases, 2018). No Brasil, observa-se o mesmo panorama, já que as doenças cardiovasculares foram responsáveis por cerca de 420 mil óbitos em 2015, com um total de 31,2\% de todas as mortes (Global Burden of Diseases, 2018). Apesar de ser a principal causa de morte no Brasil, as taxas de mortalidade das doenças cardiovasculares estão em queda nas últimas décadas, acompanhando a tendência mundial (Mansur et al., 2012), e tal redução deve-se, entre outras variáveis, ao melhor controle dos fatores de risco relacionados à doença cardiovascular aterosclerótica (Brant et al., 2017). Estima-se que o controle dos fatores de risco modificáveis contribuiu com cerca de 50\% do declínio da mortalidade observada nas últimas décadas nos países desenvolvidos (Mansur et al., 2012).

No entanto, a presença dos fatores de risco modificáveis nem sempre se traduz em doença aterosclerótica e a ausência desses, por outro lado, não garante coronárias livres de lesão (Kasliwal et al., 2014). A recomendação das diretrizes atuais, para estimativa do risco cardiovascular, é a utilização os algoritmos baseados em estudos, como o escore de Framingham, que se trata do mais utilizado (D'Agostino et al., 2008), o Systematic Coronary Risk Evaluation (SCORE) (Conroy et al., 2003), o QRESEARCH segunda versão (QRISK2) (Hippisley-Cox et al., 2008) e o escore de Reynolds (Ridker et al., 2008).

Essas são ferramentas de predição voltadas para a estimativa de risco em âmbito populacional se baseiam em fatores de risco tradicionais para as doenças cardiovasculares. Apresentam validade mais restrita na avaliação do risco individual 
(Moodakam et al., 2010), uma vez que não há relação direta entre o número de fatores de risco cardiovasculares e a carga de doença aterosclerótica individual (Lauer et al., 2007).

Por esse motivo, há crescente interesse no desenvolvimento de outras formas de avaliação de aterosclerose subclínica no âmbito individual, o que permitiria uma melhor acurácia na estimativa do risco cardiovascular de um determinado indivíduo (Greenland et al., 2001), além de possibilitar a investigação de outros potenciais fatores de risco cardiovasculares não tradicionais.

Os fatores de risco (FR) para doença cardiovascular podem ser divididos nos fatores clássicos e não clássicos. Os FR clássicos não explicam toda a carga da doença cardiovascular. Dados do Estudo Longitudinal de Saúde do Adulto (ELSA-Brasil) publicado em 2015 demonstra que os fatores de risco clássicos explicam menos de $40 \%$ do aumento de espessura da íntima-média de carótidas (EIMC), uma das medidas mais utilizadas para mensuração da aterosclerose subclínica (Santos et al., 2015). Dados internacionais explicam porcentagem ainda menor (11\%) do aumento da EIMC. Rundek et al. observaram que os fatores de risco tradicionais explicam aproximadamente $11 \%$ da variância total da EIMC. A adição de fatores de risco menos tradicionais ao modelo, como a adiponectina, aumentou essa proporção para 16\% (Rundek et al., 2013).

Além disso, apesar da sua importância já comprovada na etiologia e progressão da placa aterosclerótica, os fatores de risco cardiovasculares tradicionais não são acurados na identificação de indivíduos assintomáticos com maior risco de desenvolver a doença cardiovascular (Simon et al., 2006), sugerindo a existência de outros fatores de risco não clássicos ainda não identificados que possam também atuar na fisiopatologia da doença. 


\subsection{O estresse e o sistema cardiovascular}

A suspeita de que o estresse possa exercer um papel central no desenvolvimento de doenças cardiovasculares há muito pertence à cultura popular. A percepção do papel central que o sistema cardiovascular exerce na adaptação fisiológica ao estresse é foco de estudo desde o começo do século passado, quando Walter B. Cannon, considerado um dos fisiologistas pioneiros em emoções humanas, reuniu as primeiras evidências de que animais, quando sofriam uma experiência estressora, apresentavam uma reação a qual denominou de 'fuga ou luta'. Esta reação era decorrente da ativação do sistema nervoso simpático e que desencadeava aumento da pressão arterial e dos batimentos cardíacos, mudanças na volemia, efetividade do sistema de coagulação e disponibilidade de glicose. (Brown et al., 2002).

Atualmente, com os avanços científicos acerca das respostas fisiológicas do sistema cardiovascular ao estresse, observou-se que a reação de 'fuga ou luta' descrita por Cannon resulta de estímulos mediados tanto pelo sistema nervoso simpático e parassimpático, quanto pelo eixo hipotalâmico-pituitário-adrenal, em reação não apenas autonômica como também neuroendócrina. Tal resposta atua sobre parâmetros cardiovasculares como o débito cardíaco e a resistência vascular periférica, visando o redirecionamento do fluxo sanguíneo e a adequação da perfusão tecidual para uma eventual necessidade de esforço físico intenso iminente (Ginty et al., 2017).

A curto-prazo e como uma reação aguda, as mudanças fisiológicas do sistema cardiovascular frente ao estresse são adaptativas e conferiram vantagens evolutivas na preservação da espécie. Porém, quando prolongadas e expressas repetidamente, podem iniciar ou exacerbar alterações fisiopatológicas sobre o próprio sistema (Ginty et al., 2017). 


\subsection{Estresse relacionado ao trabalho}

A suspeita de que o estresse possa exercer um papel central no desenvolvimento das doenças cardiovasculares é amplamente discutida, mas somente nas últimas décadas, diante da competitividade entre organizações e o interesse no estudo dos fatores de risco que melhorassem o desempenho do trabalhador, surgiu uma demanda crescente por estudos que avaliassem o impacto do estresse relacionado ao trabalho sobre a saúde dos trabalhadores (Paschoal et al., 2004).

A partir dos anos 90, estudos mais sofisticados sobre estresse psicossocial no trabalho e modelos teóricos validados para defini-lo e quantificá-lo foram criados, com dois modelos principais para o estudo dos estresse associado ao trabalho: o de desequilíbrio esforço-recompensa (DER) e o da demanda-controle (DC). Ambos modelos definem diferentes estressores do ambiente laboral (Siegrist et al., 1996).

O modelo de desequilíbrio esforço-recompensa foi elaborado por Siegrist em 1996. De acordo com este modelo, recompensas, compostas por ganho financeiro, autoestima (respeito e apoio por parte de colegas e superiores) e status ocupacional (perspectivas de promoção, estabilidade no emprego e status social) poderiam moderar os efeitos negativos dos esforços relacionados ao trabalho (demandas e obrigações percebidas pelo trabalhador) em termos de sobrecarga física e psicológica. A falta de tal equilíbrio seria o determinante do estresse e do adoecimento (Siegrist et al., 1996). Posteriormente, uma terceira dimensão foi incorporada ao modelo original: o excesso de comprometimento com o trabalho. Essa seria uma dimensão intrínseca ao trabalhador, caracterizado pelo empenho exagerado no trabalho combinado a um forte desejo por aprovação, e poderia potencializar os efeitos nocivos do desbalanço entre esforço e recompensa (Griep et al., 2011). 
O segundo modelo é o modelo demanda-controle elaborado por Karasek em 1979, que considera o estresse relacionado ao trabalho como resultado de um desequilíbrio entre dois fatores essenciais do processo laboral: o controle sobre as condições de trabalho (caracterizado pela autonomia e habilidade requerida sobre o processo) e suas demandas psicológicas sobre o indivíduo (ritmo e intensidade do trabalho). Segundo Karasek, atividades com altas demandas psicológicas e baixo controle (job strain) seriam as mais propensas a favorecer o adoecimento físico e psicológico do indivíduo (Karasek et al., 1998). Pela combinação das escalas de controle do trabalho e demandas psicológicas, definem-se quatro categorias de ambiente laboral pelo modelo demanda-controle: ativo (alta demanda e alto controle), passivo (baixa demanda e baixo controle), alto desgaste ou 'job strain' (alta demanda e baixo controle) e baixo desgaste (baixa demanda e alto controle) (Alves et al., 2004).

Assim como ocorrido com o modelo de esforço-recompensa, o modelo demandacontrole passou, a partir de 1988, a incluir uma terceira dimensão, elaborada por Johnson e Hall: a percepção pelo trabalhador de apoio ou integração social. Segundo os autores, a interação com a equipe, tanto como parte da execução do trabalho como pela integração em ambientes externos ao laboral, poderia minimizar os efeitos nocivos do desgaste laboral sobre a saúde do trabalhador (Johnson et al., 1988).

Originalmente, o instrumento criado por Karasek para avaliar as características psicológicas e sociais do trabalho foi o Job Content Questionnaire (JCQ), incluindo 49 questões abrangendo as dimensões do modelo demanda-controle e incluindo outras variáveis, como demandas físicas e percepção de insegurança pelo trabalhador (Karasek et al., 1998). Posteriormente, em ação colaborativa de diversos pesquisadores de vários países do mundo, surgiram novas adaptações, diferentes quanto ao número de itens, formato de resposta ou conteúdo dos itens, porém consistentes com o modelo teórico 
demanda-controle, como o questionário desenvolvido por Theorell na Suécia (Theorell et al., 1988), pelo Estudo Whitehall na Inglaterra (Kuper et al., 2003) e pelo Estudo MONICA na Dinamarca (Karasek et al., 2008; Alves et al., 2013).

No Brasil, existem dois diferentes instrumentos traduzidos e adaptados para o português brasileiro para aplicação do modelo demanda-controle: o Job Content Questionnaire (JCQ) (de Araújo, 2008) e a 'Escala Sueca de Demanda-Controle-Apoio Social' (DCS) (Theorell et al., 1996; Griep et al., 2011).

A 'Escala Sueca de Demanda-Controle-Apoio Social' (DCS) é uma versão reduzida do questionário originalmente elaborado por Karasek, contendo 17 questões com enfoque nas três dimensões do modelo DC. Alves et al. realizaram a adaptação da versão sueca para o português e, para avaliação no nível de estabilidade das respostas, realizaram um estudo de confiabilidade teste-reteste com 94 indivíduos selecionados, estimando coeficientes de correlação intraclasse para as dimensões de 'demanda', 'controle' e 'apoio social' da escala de 0,88, 0,87 e 0,85, respectivamente. Para avaliação da consistência interna das perguntas, foram estimados coeficientes alpha de Cronbach para cada uma das dimensões 'demanda', 'controle' e 'apoio social' entre os participantes do Estudo Pró-Saúde, uma coorte com 3.574 funcionários técnico-administrativos do Rio de Janeiro, com valores respectivamente de 0,79, 0,67 e 0,85 (Alves et al., 2004).

Aguiar e colaboradores, utilizando-se da Escala DCS e visando a análise de aplicabilidade em uma população de baixa escolaridade, também procederam com um estudo de confiabilidade teste-reteste, aplicando o questionário para 52 trabalhadores de restaurantes industriais do Rio de Janeiro, em dois momentos distintos. Para as dimensões ‘demanda psicológica', 'controle do trabalho' e 'apoio social', Aguiar et al. encontraram coeficientes de correlação intraclasse de 0,70, 0,68 e 0,80, e coeficientes alpha de Cronbach de 0,75, 0,50 e 0,82, respectivamente. Os autores concluem que, ainda que os 
resultados de consistência interna referentes ao controle do trabalho tenham sido baixos, os outros indicadores de confiabilidade apontam para uma boa estabilidade do instrumento, sugerindo um processo bem-sucedido de adaptação da escala para o contexto sociocultural brasileiro (Aguiar et al., 2010).

No presente estudo, optamos por utilizar a escala Demanda-Controle-Social (DCS), em sua versão traduzida para o português por Alves et al. (Alves et al., 2013), que se baseia no modelo atualizado de Karasek, após inclusão de sua terceira dimensão de análise, o apoio social.

\subsection{Medida da aterosclerose subclínica}

A aterosclerose é uma doença inflamatória crônica e progressiva com um longo período assintomático precedendo o evento cardiovascular e, durante este período, o manejo dos fatores de risco cardiovasculares pode minimizar ou mesmo prevenir desfechos cardiovasculares (Toth, 2008). O método mais empregado atualmente para predição do risco cardiovascular é o escore de risco de Framingham, um algoritmo desenvolvido com base no estudo Framingham Heart Study e que estima, utilizando-se de idade, gênero e fatores de risco cardiovasculares tradicionais, o risco de desenvolvimento de doença arterial coronariana em 10 anos (Wilson et al., 2014).

No entanto, a predição de risco cardiovascular com base em algoritmos apresenta limitações. Algumas populações não apresentam acurácia discriminativa quando estimado o risco cardiovascular, como mulheres e indivíduos mais jovens, que tendem a ser classificados como baixo risco (Sibley et al., 2002; Berry et al., 2009). Outra limitação de relevância refere-se à grande proporção de eventos cardiovasculares que ocorrem em indivíduos não considerados como alto risco cardiovascular (Grover et al., 1995). Khot 
et al., em uma metanálise com 122.458 indivíduos que utilizou 14 estudos internacionais sobre doença coronariana, avaliaram a prevalência dos quatro fatores de risco cardiovasculares considerados tradicionais para doença cardiovascular - tabagismo, diabetes, dislipidemia e hipertensão arterial. Em pacientes com doença coronariana aguda, angina instável ou com necessidade de intervenção coronariana percutânea foi observado que $10,7 \%$ das mulheres e $13,3 \%$ dos homens entre 46 e 55 anos não apresentavam nenhum dos fatores de risco cardiovasculares considerados tradicionais (Khot et al., 2003).

A estimativa da carga de doença aterosclerótica individual mostrou-se um método de rastreamento eficaz na predição de eventos cardiovasculares futuros (Fernández-Friera et al., 2014). Simon et al., em uma análise de seis estudos prospectivos incluindo 16.352 indivíduos sem doença cardiovascular prévia e assintomáticos, avaliaram marcadores de aterosclerose subclínica por meio da espessura íntima-média de carótidas, do escore de cálcio em artérias coronárias, do índice tornozelo-braquial e da velocidade de onda de pulso carotídeo-femoral, e calcularam a incidência de evento coronariano em um período de um a sete anos de seguimento. De acordo com a análise, valores de espessura íntimamédia carotídeas acima do percentil 95 foram associados a 1,2 a 1,6\% de incidência anual de doença coronariana, enquanto indivíduos abaixo deste percentil tiveram $<0,5 \%$ de incidência anual; escores de cálcio de 400 Agatston foram associados a 3,3\% de incidência anual de doença coronariana aguda, enquanto escores igual a zero apresentaram incidência menor do que $0,6 \%$; índice tornozelo-braquial $<0,9$ foram associados a 1,6\% de incidência anual de doença coronariana, enquanto indivíduos com valores $>0,9$ apresentaram $<1 \%$ de incidência; e indivíduos com velocidade de onda de pulso carotídeo-femoral acima do terceiro tercil foram associados a 1,4\% de incidência de doença coronariana ao ano, em contraste a incidência de $0,4 \%$ em pacientes com 
velocidade de onda de pulso no primeiro tercil usado como a referência (Simon et al., 2006).

Existem exames de imagens não invasivos para avaliar a aterosclerose subclínica, mas a aferição da deposição de cálcio nas artérias coronárias (CAC) por meio de tomografia computadorizada e a mensuração da espessura íntima-média de carótida (EIMT) são os métodos com maior evidência científica para avaliação de risco cardiovascular individual (Moodakam et al., 2010).

\subsection{Espessura Íntima-Média de Carótidas}

A medida da espessura das camadas íntima e média das artérias carótidas, denominada 'espessura íntima-média da carótida' (EIMC), vem sendo pesquisada extensivamente há duas décadas e tem sido utilizada principalmente para pesquisa, embora esteja disponível no contexto médico desde 2002. Trata-se de método nãoinvasivo, de baixo custo e boa reprodutibilidade (Kasliwal et al., 2014).

Convencionalmente, a ultrassonografia com doppler das artérias carótidas sem a estimativa da EIMC é utilizada na prática clínica para identificar placas oclusivas em seu trajeto, sendo uma manifestação da aterosclerose avançada. Para acessar o risco cardiovascular, a EIMC avalia a parede das artérias carotídeas ao invés do grau de estreitamento luminal, com foco na identificação de áreas de aumento de espessura e de placas ateroscleróticas não oclusivas, que representam estágios precoces de lesão arterial e aterosclerose subclínica (Stein et al., 2008). 
Aumentos na EIMC ocorrem com o avanço da idade, mesmo na ausência de aterosclerose, relacionada à hipertrofia intimal, medial ou ambas, configurando-se como uma resposta adaptativa a mudanças no fluxo sanguíneo, tensão na parede vascular ou diâmetro luminal (Bots et al., 1997). Essas alterações abrangem diversos fatores implicados na patogênese e progressão das placas ateroscleróticas, como disfunção endotelial; aumento na aderência e permeabilidade das células endoteliais; aumento de moléculas vasoconstritoras, pró-coagulantes e pró-inflamatórias; aumento nas citocinas; aumento no estresse oxidativo; e proliferação e migração de células musculares lisas (Li et al., 1999; Asai et al., 2000). Portanto, o espessamento médio-intimal é um atributo evolutivo da parede arterial que não se constitui como sinônimo de aterosclerose subclínica, mas está diretamente relacionado à aterosclerose subclínica, pois as alterações celulares e moleculares no espessamento da íntima-média são substrato fisiopatológico importante para o desenvolvimento e progressão da aterosclerose.

Existem diversos estudos prospectivos sobre a medida da EIMC e o risco futuro de eventos cardiovasculares que mostraram sua associação com aumento do risco de doença coronariana. (Simon et al., 2006; Rosvall et al., 2005; Chambless et al., 1997). Rosvall et al. realizaram um estudo de coorte com 5163 participantes sem eventos isquêmicos prévios e com seguimento de sete anos, e observaram que o incremento de 0,15 mm de EIMC está associado a razão de risco de eventos coronarianos de 1,23 (Intervalo de Confiança a 95\% (IC 95\%), 1,07-1,41) (Rosvall et al., 2005). No estudo The Atherosclerosis Risk in Communities Study (ARIC) realizado por Chambless et al., foram avaliados 12841 indivíduos entre 45 e 64 anos e livres de doença cardiovascular prévia por meio da EIMC, e analisado o risco prospectivo de doença coronariana durante um seguimento de 4 a 7 anos. Quando comparadas EIMC consideradas 'extremas' ( $\geq 1 \mathrm{~mm})$ com 'não-extremas' (<1mm), os autores observaram que mulheres apresentaram a razão 
de risco de 5,07 (IC 95\%, 3,08-8,36) e homens apresentaram razão de risco de 1,85 (IC 95\%, 1,28-2,69) para eventos coronarianos (Chambless et al., 1997).

Além da associação entre EIMC e eventos coronarianos, diversos autores realizaram estudos para avaliar a EIMC como preditora de acidente vascular cerebral, tanto isoladamente como em desfechos combinados (Rujiter et al., 2012). Ruijter et al. realizaram uma metanálise de 14 estudos de coorte prospectivos, incluindo 45.858 indivíduos e com seguimento médio de 11 anos. Foram analisados 4007 novos eventos e observado que, a cada $0,1 \mathrm{~mm}$ de incremento na EIMC, houve associação com razão de risco de 1,08 (IC 95\%, 1,05-1,10) para infarto agudo do miocárdio e razão de risco de 1,12 (IC 95\%, 1,10-1,15) para acidente vascular cerebral (Rujiter et al., 2012).

\subsection{Tomografia Computadorizada com Mensuração de Cálcio de Coronárias}

Mais recentemente, a tomografia computadorizada para a mensuração de cálcio nas artérias coronárias (CAC) vem sendo considerada uma nova ferramenta de rastreamento não invasivo de aterosclerose subclínica e preditora de eventos cardiovasculares futuros, com resultados superiores aos da EIMC (Folsom et al., 2008). A CAC infere a presença de aterosclerose subclínica medindo a quantidade de depósito de cálcio nas artérias coronárias e apresenta uma boa correspondência com o volume de placas neste território como demonstrado em um estudo post-mortem (Rumberger et al., 1995).

Deltrano et al. avaliaram 6722 adultos inicialmente assintomáticos com base nos dados do Multi-Ethnic Study of Atherosclerosis (MESA), uma das primeiras grandes coortes que incluiu a medida da CAC como parte do seu protocolo de exames, com 
mediana de seguimento de 3,8 anos e observaram incremento no valor prognóstico proveniente da utilização da CAC, pois participantes com escore de cálcio de 1 a 100 apresentaram razão de risco de 3,89 (IC 95\%, 1,72-8,79) para infarto agudo do miocárdio ou morte por doença coronariana; participantes com CAC 101-300 apresentaram razão de risco 7,08 (IC 95\%, 3,05-16,47); e participantes com CAC acima de 300 apresentaram razão de risco 6,84 (IC 95\%, 2,93-15,99) (Deltrano et al., 2008).

Elias-Smale et al. estudaram 2028 indivíduos de mais de 55 anos e estratificaram conforme o risco cardiovascular tanto pelo escore de Framingham quanto pela CAC. Eles concluíram pela eficácia semelhante de ambas as estratégias nos subgrupos de baixo e alto risco cardiovascular, porém enfatizaram a importância da CAC na reclassificação dos indivíduos categorizados no grupo de risco intermediário pelo Escore de Framingham utilizando-se o CAC (Elias-Smale et al., 2010).

\subsection{Estresse no trabalho e aterosclerose subclínica}

Uma revisão sistemática de 2014 listou os estudos que avaliaram estresse no trabalho e EIMC (Wilson et al., 2014). A revisão encontrou vinte e nove estudos, mas 20 entre eles eram duplicados ou não preencheram os critérios de seleção. Um último estudo foi encontrado nas referências dos previamente selecionados, totalizando dez estudos. Dos dez estudos selecionados, seis eram transversais e quatro eram prospectivos. Oito estudos encontraram uma associação positiva entre estresse no trabalho e EIMC (Eller et al., 2007; Everson et al., 1997; Hintsa et al., 2008; Kamarck et al., 2012; Hintsane et al., 2008; Nordstrom et al., 2001; Rosvall et al., 2002; Xu et al., 2010). Jedryka-Goral et al. relatou uma associação inversa estatisticamente significativa entre estresse no trabalho e EIMC (Jedryka-Goral et al., 2006). Fujishiro et al., encontraram somente uma associação 
entre controle no trabalho e EIMC (Fujishiro et al., 2011). A amostra nesses estudos variou de 94 a 24.801 indivíduos, com média de seguimento de 4 a 6 anos. A maioria dos estudos foi financiado por agências do governo, e em uma avaliação do risco de viés nesses estudos foi considerada como baixa. Analisando os resultados encontrados por tipo de desenho do estudo, verificou-se que os quatro estudos de coorte prospectivos mostraram associação de estresse no trabalho com aumento da EIMC. Três estudos de coorte usaram o modelo demanda-controle. Cinco dos seis estudos mostraram uma associação positiva entre estresse no trabalho e EIMC. O estudo de Jedryka-Goral encontrou uma associação entre controle no trabalho e aumento de EIMC (Jedryka-Goral et al., 2006). Os autores encontraram presença de viés de publicação com muito mais estudos publicados com resultados positivos e poucos com resultados negativos.

Somente um estudo avaliou estresse no trabalho e aterosclerose subclínica avaliado pelo escore de cálcio. No the Coronary Artery Risk Development in Young Adults (CARDIA) study, o CAC foi medido em 2000 e 2001 e reavaliado entre 2005 e 2006. As características do trabalho foram mensuradas pelo modelo demanda-controle incluindo demandas psicológicas e autoridade decisória em 1987 e 1988 e posteriormente em 1995 e 1996. Os resultados mostraram que a autoridade decisória e o estresse no trabalho não se associaram com a presença de CAC (Greenlund et al., 2010).

Desta maneira, se faz necessário realizar mais estudos avaliando a associação entre estresse laboral e medidas de aterosclerose subclínica, inclusive em novos contextos como o Brasil. 


\section{Objetivos}

\subsection{Objetivo principal}

Avaliar a associação entre estresse no trabalho e aterosclerose subclínica mensurada pelo Escore de Cálcio (CAC) e pela Espessura Íntima-Média de Carótidas (EIMC)

\subsection{Objetivos secundários}

Avaliar essa associação usando a apresentação clássica de demanda, controle e apoio social e subdividindo-se o controle em uso de habilidades e autoridade decisória.

Avaliar o efeito do uso de pontos de corte diferenciados para a medida de Escore de Cálcio (CAC $>0$ Agatston e $\mathrm{CAC} \geq 100$ Agatston).

Avaliar o efeito do uso de ponto de corte da EIMC (EIMC Percentil $>75 \%$ ) e como variável contínua

\subsection{Hipótese}

Estresse no trabalho se associa com aterosclerose subclínica medida pelo CAC e pela EIMC. 


\section{Métodos}

O Estudo Longitudinal de Saúde do Adulto (ELSA-Brasil) é um estudo de coorte prospectivo com 15.105 funcionários públicos com idades entre 35 e 74 anos de seis instituições de ensino e pesquisa no país: Universidade Federal da Bahia (UFBA), Universidade Federal de Minas Gerais (UFMG), Universidade Federal do Rio Grande do Sul (UFRGS), Fundação Oswaldo Cruz (Fiocruz, RJ) e Universidade de São Paulo (USP). Todos os funcionários dessas instituições na faixa etária requerida pelo estudo eram elegíveis. Os critérios de inelegibilidade foram: gravidez atual ou menos de 4 meses entre o parto e a coleta de dados do estudo, intenção de sair do emprego em futuro próximo, dificuldade de comunicação ou alteração cognitiva importante e residência fora da zona metropolitana onde se localiza o Centro de Investigação do estudo. A coleta de dados da linha de base do estudo ocorreu entre Agosto de 2008 e Dezembro de 2010. Na linha de base foram aplicados questionários, realizada coleta de sangue e urina e vários exames como eletrocardiograma, velocidade de onda de pulso e variabilidade da frequência cardíaca. (Aquino et al., 2012; Schmidt et al., 2015; Benseñor et al., 2013).

Esta é uma análise transversal feita com dados da linha de base em 4549 participantes do Centro de Investigação de São Paulo que foram submetidos ao escore de cálcio (CAC) como parte das avaliações realizadas na linha de base. 


\subsection{Estresse no trabalho}

O modelo de estresse no trabalho avaliado na linha de base do ELSA-Brasil foi detalhado em publicações prévias (Santos et al., 2014; Camelo et al., 2015) O estudo utilizou a versão validada para o português do Brasil do 17-Item Swedish DemandControl-Support Questionnaire (SDSQ) (Alves et al., 2004) que se baseia no The Job Content Questionnaire elaborado por Karasek e colaboradores em 1998 (Karasek et al., 1998). Robert Karasek foi um dos pesquisadores pioneiros a procurar nas relações sociais do ambiente de trabalho fontes geradoras de estresse e suas repercussões sobre a saúde. Nos anos 70, propôs um modelo teórico bidimensional que relacionava dois aspectos demanda e controle no trabalho - ao risco de adoecimento. $\mathrm{O}$ foco do modelo de Karasek encontra-se no modo de organização do trabalho. As demandas são pressões de natureza psicológica, sejam elas quantitativas, tais como tempo e velocidade na realização do trabalho, ou qualitativas, como os conflitos entre demandas contraditórias. O controle é a possibilidade do trabalhador utilizar suas habilidades intelectuais para a realização de seu trabalho, bem como possuir autoridade suficiente para tomar decisões sobre a forma de realizá-lo (Theorell et al., 1996; Theorell, 2000). Para definir estresse no trabalho seguindo o modelo de Karasek, os escores das subescalas de demanda e controle devem ser dicotomizados como valores baixos ( $\leq$ mediana) versus valores altos (> mediana). A partir dessa definição são criados quatro grupos mutuamente exclusivos classificando os participantes. (Figura 1). A coexistência de grandes demandas psicológicas com baixo controle sobre o processo de trabalho gera o alto desgaste ("job strain") no trabalhador, com efeitos nocivos à sua saúde. A combinação de baixas demandas e baixo controle também é nociva (trabalho passivo), na medida em que gera perda de habilidades e desinteresse. Por outro lado, quando altas demandas e alto controle coexistem, os 
indivíduos experimentam o processo de trabalho de forma ativa: ainda que as demandas sejam excessivas, elas são menos danosas, na medida em que o trabalhador pode escolher como planejar suas horas de trabalho de acordo com seu ritmo biológico e criar estratégias para lidar com suas dificuldades (Theorell et al., 1996). A situação "ideal", é a que combina baixas demandas e alto controle do processo de trabalho denominada de baixo desgaste.

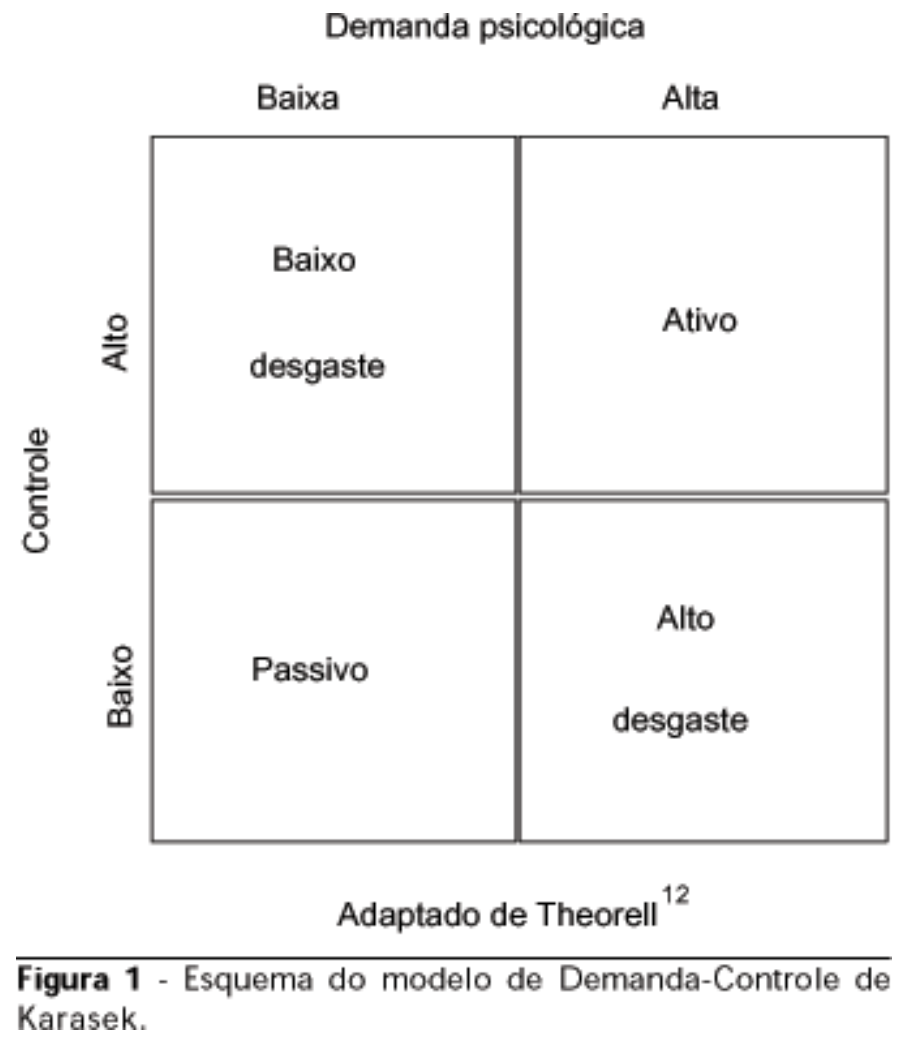

A escala tem uma terceira dimensão que é o apoio social no ambiente de trabalho. Essa dimensão foi acrescentada ao modelo por Johnson, em 1988 (Johnson et al., 1988), e é definida por seus autores como os níveis de interação social, existentes no trabalho, tanto com os colegas quanto com os chefes. Sua escassez também pode gerar 
consequências negativas à saúde. A versão brasileira do DCSQ apresentou coeficientes de correlação intraclasse de de 0.88, 0.87 e 0.86; e alfa de Cronbach de 0.72, 0.63 e 0,86 respectivamente para os domínios demanda, controle e suporte social (Alves et al., 2004).

Uma análise mais detalhada da estrutura fatorial e da invariância ao longo do tempo realizada por Chungkham et al. mostrou que os domínios de uso de habilidades e autoridade decisória são pouco correlacionados refletindo aspectos diferentes do estresse no trabalho. Além disso, essas analises sugeriram que a questão sobre trabalho repetitivo deveria ser retirada. (Chungkham et al., 2013) A análise apresentada nesta tese incorporou essas modificações. Os participantes foram classificados para cada um desses domínios em três grupos de tamanho similar (escore baixo, moderado e alto) de acordo com a tabela abaixo. Essa estratégia de análise já foi adotada em outros estudos do ELSA-Brasil (Santos et al, 2014; Camelo et al., 2015).

Nesta tese, os resultados serão apresentados das duas formas: o modelo clássico em 4 domínios e o novo modelo com demanda, uso de habilidades e autoridade decisória 
Quadro 1. Amplitude e classificação dos domínios do estresse no trabalho.

\begin{tabular}{lcccc}
\hline $\begin{array}{l}\text { Domínio de } \\
\text { estresse no }\end{array}$ & $\begin{array}{c}\text { Amplitude } \\
\text { (pontos) }\end{array}$ & Baixo (pontos) & $\begin{array}{c}\text { Médio } \\
\text { (pontos) }\end{array}$ & Alto (pontos) \\
trabalho & & & & \\
\hline Demanda & $5-20$ & $5-12$ & $13-15$ & 12 \\
\hline Uso de & $3-12$ & $3-9$ & $10-11$ & 8 \\
habilidades & & & & \\
\hline Autoridade & $2-8$ & $2-5$ & $6-7$ & \\
decisória & $6-24$ & $6-19$ & $20-22$ & \\
\hline Suporte Social & & & & \\
\hline
\end{tabular}

\subsection{Mensuração da aterosclerose subclínica}

\subsubsection{Escore de cálcio em artérias coronárias}

O escore de cálcio em coronárias foi medido em uma subamostra de participantes ELSA-Brasil do centro de investigação de São Paulo na linha de base. Foram realizados 4549 exames de CAC na linha de base. Para a medida do escore de cálcio em coronárias (CAC) foram utilizados dois tomógrafos Philips Brilliance com 64 canais disponíveis no Hospital Universitário da USP. O CAC foi calculado pelo método de Agatston. Para 
aquisição da imagem o exame foi sincronizado com o eletrocardiograma (ECG), para a reconstrução da imagem durante o período da diástole, que é o período de menor movimento e menor risco de artefatos na aquisição da imagem. Posteriormente, as imagens foram processadas e laudadas por cardiologistas com experiência no método, conforme padronização reconhecida na literatura. O CAC foi mensurado em Unidades Agatston usando um software semiautomático (Calcium Scoring, Philips Workstation). O escore de cálcio em coronárias foi categorizado como 0 ou $>0,>100$ ou $\geq 100$. (Pereira et al., 2016; Agatston et al., 1990)

\subsubsection{Espessura íntima-média de carótidas}

A técnica para realização da EIMC no ELSA-Brasil foi publicada previamente (Mill et al., 2013; Santos et al., 2014). O mesmo protocolo foi executado em todos os centros usando um aparelho de ultrassonografia Toshiba Aplio $\mathrm{XG}^{\mathrm{TM}}$ com transdutor linear de 7.5 MHz. A EIMC foi mensurada na artéria carótida comum, na parede mais distante do técnico em um segmento de $1 \mathrm{~cm}$ pré-definido, distante $1 \mathrm{~cm}$ da bifurcação das carótidas e durante três ciclos cardíacos. As imagens obtidas foram enviadas para o centro de leitura centralizada de São Paulo. Utilizou-se o software MIATM (Medical $^{\text {The }}$ Imaging Applications, Coralville, Iowa, USA) para padronização da leitura. Nesta análise será utilizada a média dos valores médios e dos valores máximos de EIMC das artérias carótidas direita e esquerda. A EIMC foi utilizada como variável contínua e como variável categórica classificada como EIMC $\leq$ Percentil 75\% ou $>$ Percentil 75\%. 


\subsection{Demais variáveis}

Este estudo incluiu as seguintes variáveis sociodemográficas: idade (em anos), sexo (masculino e feminino), auto relato de raça (branca, parda, preta, oriental e indígena), escolaridade (fundamental completo e incompleto, curso médio e superior incompleto, pelo menos superior completo), renda familiar mensal média $(\leq \mathrm{R} \$ 2.590,00$, $\mathrm{R} \$ 2591,00$ a $\mathrm{R} \$ 6.637,00$ e $\geq \mathrm{R} \$ 6638,00$ ), ter um plano de saúde privado (sim ou não).

As medidas antropométricas incluíram o índice de massa corpórea (IMC) calculado como peso dividido pela altura ao quadrado (em metros) e expresso em $\mathrm{kg} / \mathrm{m}^{2}$ e a medida da circunferência da cintura expressa em $\mathrm{cm}$ usando protocolos padronizados iguais nos seis centros. Hipertensão arterial foi definida como o uso de medicações para tratamento da hipertensão ou pressão arterial sistólica $140 \mathrm{mmHg}$ ou pressão arterial diastólica $\geq 90 \mathrm{mmHg}$. Diabetes foi definido como história médica prévia de diabetes, uso de medicação para tratamento do diabetes ou se a glicemia de jejum for superior a 125 ou se a glicemia após 2 horas for $\geq 140 \mathrm{mg}$ ou se a hemoglobina glicada $\geq 6.5 \%$. Dislipidemia foi definida como o uso de tratamento para diminuir os níveis de colesterol e triglicérides ou LDL-colesterol > $130 \mathrm{mg}$. Tabagismo foi definido como nunca, passado ou atual. Ingestão de álcool foi definida como nunca, passada ou atual. (Benseñor et al., 2013; Fedeli et al., 2013). Atividade física no lazer foi classificada de acordo com os critérios da Organização Mundial da Saúde usando o International Physical Activity Questionnaire (IPAQ), categorizando como ativo participantes com pelo menos 150 minutos de atividade física de intensidade moderada ou 75 minutos de atividade física intensa ou uma combinação de ambas; Qualquer prática de atividade física inferior a esse 
nível foi classificada como insuficientemente ativo e os participantes que não praticam atividade física como sedentários (IPAQ, 2017).

\subsection{Análise estatística}

As variáveis categóricas estão apresentadas na forma de números absolutos e proporções e comparada pelo teste do qui-quadrado. Variáveis contínuas foram apresentadas como média \pm desvio-padrão ou mediana (intervalo interquartil) e comparadas usando-se o teste de Mann-Whitney ou Kruskall-Wallis conforme necessário categorizados em tercis como baixo, médio e alto.

Modelos de regressão logística foram construídos usando os domínios do estresse no trabalho como as variáveis independentes e o CAC e a EIMC como as variáveis dependentes. Para demanda a referência foi a categoria baixa e para uso de habilidades e autoridade decisória (controle) e suporte social a referência ficou na categoria alta. As Razões de Chance e respectivos Intervalos de Confiança a 95\% foram apresentadas na forma sem ajuste, ajustada por fatores sociodemográficos como idade, sexo, raça autodeclarada e ajuste multivariado incluindo as variáveis sociodemográficas do modelo 1 mais o IMC e os fatores de risco para doença cardiovascular como hipertensão, diabetes, dislipidemia, tabagismo, consumo de álcool e prática atividade física. Todas as análises foram feitas usando o software Social Package for Social Sciences, versão 22 (SPSS Inc., Chicago, Illinois, USA) e o valor de $\mathrm{p}<0.05$ estatisticamente significativa. 


\subsection{Aspectos Éticos}

O protocolo do estudo seguiu as resoluções da Declaração de Helsinki de 1975 sobre pesquisa em seres humanos. O projeto foi aprovado nos Comitês de Ética em Pesquisa dos seis centros. Todos os participantes assinaram consentimento informado. (Aquino et al., 2013) 


\section{Resultados}

A análise incluiu somente os participantes de São Paulo que foram submetidos ao CAC na linha de base. Dos 5061 participantes de São Paulo, 4549 fizeram o CAC na linha de base. Dentre eles, foram excluídos da análise 749 participantes que não tinham informações sobre estresse no trabalho por serem aposentados, 258 que tinham história prévia de doença cardiovascular e 136 que não tiveram a EIMC mensurada na linha de base, restando 3.490 participantes que foram incluídos na análise. Desses, 1832 (52,9\%) eram mulheres.

A Tabela 1 descreve as características gerais dos participantes de acordo com o sexo. Exceto por idade e índice de massa corpórea (IMC) que não apresentam diferenças entre os sexos, todas as outras variáveis são diferentes. As mulheres são mais educadas do que os homens $(\mathrm{P}<0,0001)$. Também apresentam frequências mais baixas de hipertensão, diabetes, dislipidemia em relação aos homens. ( $\mathrm{P}<0.0001$ para hipertensão

e diabetes e $\mathrm{P}=0,03$ para dislipidemia). A porcentagem de nunca fumantes e de nenhum consumo de álcool são mais elevadas nas mulheres em relação aos homens ( $\mathrm{P}<0,0001$ para as duas variáveis). 
Tabela 1. Características gerais da amostra de acordo com o sexo.

\begin{tabular}{|c|c|c|c|}
\hline & \multirow{2}{*}{$\begin{array}{l}\text { Homens } \\
\mathrm{N}=1643\end{array}$} & \multirow{2}{*}{$\begin{array}{l}\text { Mulheres } \\
\mathrm{N}=1847\end{array}$} & \multirow[t]{2}{*}{$\mathrm{P}$} \\
\hline & & & \\
\hline Idade média (DP)* & $48,6(7,2)$ & $48,2(6,8)$ & 0,08 \\
\hline Raça (\%) & & & $<0,0001$ \\
\hline Branca & $912(56,4)$ & $1094(59,7)$ & \\
\hline Parda & $419(25,9)$ & $376(20,5)$ & \\
\hline Preta & $223(13,8)$ & $264(14,4)$ & \\
\hline Amarela & $44(2,7)$ & $87(4,7)$ & \\
\hline Indígena & $20(1,2)$ & $11(0,6)$ & \\
\hline Educação (\%) & & & $<0,0001$ \\
\hline Menos que Ensino médio & $304(18,5)$ & $156(8,4)$ & \\
\hline Ensino médio e superior incompleto & $730(44,4)$ & $790(42,8)$ & \\
\hline Superior completo ou mais & $609(37,1)$ & $901(48,9)$ & \\
\hline Índice de massa corpórea $\mathrm{kg} / \mathrm{m}^{2}$ (DP) & $27,1(4,4)$ & $27,1(5,1)$ & 0,99 \\
\hline Hipertensão (\%) & $512(31,2)$ & $395(21,4)$ & $<0,0001$ \\
\hline Diabetes $(\%)$ & $330(20,1)$ & $241(13)$ & $<0,0001$ \\
\hline Dislipidemia (\%) & $923(56,8)$ & $955(51,8)$ & 0,003 \\
\hline Tabagismo (\%) & & & $<0,0001$ \\
\hline Nunca & $806(49,1)$ & $1098(59,4)$ & \\
\hline Passado & $555(33,8)$ & $470(25,4)$ & \\
\hline Atual & $282(17,2)$ & $279(15,1)$ & \\
\hline Ingestão alcoólica (\%) & & & $<0,0001$ \\
\hline Nunca & $76(4,6)$ & $322(17,4)$ & \\
\hline Passada & $344(20,9)$ & $350(18,9)$ & \\
\hline Atual & $1223(74,4)$ & $1175(63,6)$ & \\
\hline Atividade física (\%) & & & $<0,0001$ \\
\hline Leve & $1192(75,7)$ & $1464(82,4)$ & \\
\hline Moderada & $217(13,8)$ & $191(10,8)$ & \\
\hline Vigorosa & $166(10,5)$ & $121(6,8)$ & \\
\hline
\end{tabular}

*Média (DP) 
A Tabela 2 mostra as médias de aterosclerose subclínica na amostra por sexo.

A frequência de $\mathrm{CAC}>0, \geq 100 \mathrm{e} \geq 400$ foi mais elevada em homens do que nas mulheres. Os valores médios de EIMC também foram mais altos nos homens do que nas mulheres assim como a frequência de EIMC acima do P75\%. A mediana de demanda foi mais elevada nas mulheres do que nos homens e a mediana do suporte social foi mais elevada nos homens do que nas mulheres, sem diferenças nos valores da mediana entre uso de habilidades e autoridade decisória. A distribuição de estresse no trabalho pelo modelo de Karasek foi diferente em homens e mulheres. Os homens apresentaram frequências mais elevadas de alto desgaste e comportamento passivo enquanto as mulheres apresentaram frequências mais elevadas de comportamento ativo e alto desgaste. 
Tabela 2. Nível de aterosclerose subclínica e dos domínios de estresse no trabalho de acordo com o sexo

\begin{tabular}{|c|c|c|c|}
\hline & $\begin{array}{l}\text { Homens } \\
\mathrm{N}=1643\end{array}$ & $\begin{array}{l}\text { Mulheres } \\
\mathrm{N}=1847\end{array}$ & \\
\hline CAC (Mediana, Intervalo & $0(0-12,8)$ & $0(0-91,9)$ & $<0,0001$ \\
\hline \multicolumn{4}{|l|}{ Interquartil) $\mathrm{CAC}$} \\
\hline $\mathrm{CAC}>0(\%)$ & $570(34,7)$ & $241(13)$ & $<0,0001$ \\
\hline $\mathrm{CAC} \geq 100(\%)$ & $190(11,6)$ & $52(2,8)$ & $<0,0001$ \\
\hline $\mathrm{CAC} \geq 400(\%)$ & $62(3,8)$ & $11(0,6)$ & $<0,0001$ \\
\hline EIMC média das médias mm (DP) & $0,60(0,13)$ & $0,57(0,11)$ & $<0,0001$ \\
\hline EIMC média dos máximos mm (DP) & $0,75(0,16)$ & $0,71(0,14)$ & $<0,0001$ \\
\hline EIMC média das médias >P75 (\%) & $498(30,3)$ & $344(18,6)$ & $<0,0001$ \\
\hline $\begin{array}{l}\text { EIMCT média dos máximos >P75 } \\
(\%)\end{array}$ & $484(29,5)$ & $355(19,2)$ & $<0,0001$ \\
\hline \multicolumn{4}{|l|}{ Domínios do estresse no trabalho } \\
\hline \multicolumn{4}{|l|}{ Interquartil) } \\
\hline \multicolumn{3}{|l|}{ Intervalo Interquartil) } & 0,02 \\
\hline $\begin{array}{l}\text { Autoridade decisória (mediana, } \\
\text { Intervalo Interquartil) }\end{array}$ & $6(5-7)$ & $6(5-7)$ & 0,15 \\
\hline Suporte Social (mediana, Intervalo & $20(18-23)$ & $20(17-22)$ & $<0,0001$ \\
\hline \multicolumn{4}{|l|}{ Interquartil) } \\
\hline Estresse no trabalho pelo modelo de & & & $<0,0001$ \\
\hline \multicolumn{4}{|l|}{ Karasek } \\
\hline Baixo desgaste & $317(19,3)$ & $264(14,3)$ & \\
\hline Passivo & $611(37,2)$ & $589(31,9)$ & \\
\hline Ativo & $313(19,1)$ & $395(21,4)$ & \\
\hline Alto desgaste & $402(24,5)$ & $599(32.4)$ & \\
\hline
\end{tabular}

*DP = desvio-padrão; **IIQ = Intervalo Interquartil; $†$ CAC $=$ Escore de cálcio $;$ †EIMC = Espessura íntima-média de carótidas. 
A Tabela 3 mostra as características sociodemográficas e clínica dos participantes de acordo com as categorias de estresse no trabalho. O comportamento passivo foi o mais frequente seguido pelo alto desgaste, comportamento ativo e baixo desgaste. 
Tabela 3. Características gerais dos participantes de acordo com as categorias de estresse no trabalho.

\begin{tabular}{|c|c|c|c|c|c|}
\hline & $\begin{array}{l}\text { Baixo desgaste } \\
\mathrm{N}=581\end{array}$ & $\begin{array}{l}\text { Passivo } \\
\mathrm{N}=1200\end{array}$ & $\begin{array}{l}\text { Ativo } \\
\mathrm{N}=708\end{array}$ & $\begin{array}{l}\text { Alto desgaste } \\
\mathrm{N}=1001\end{array}$ & $\mathrm{P}$ \\
\hline Idade média (DP*) & $49,9(7,8)$ & $48,1(6,7)$ & $49,4(7,3)$ & $47,0(6,3)$ & $\begin{array}{l}<0,0001 \\
<0,0001 * *\end{array}$ \\
\hline Mulheres (\%) & $264(45,4)$ & $589(49,1)$ & $395(55,8)$ & $599(59,8)$ & $<0,0001$ \\
\hline \multicolumn{6}{|l|}{ Raça (\%) } \\
\hline Branca & $409(71,1)$ & $615(51,7)$ & $473(68)$ & $509(51,4)$ & $<0,0001$ \\
\hline Parda & $93(16,2)$ & $341(28,7)$ & $96(13,8)$ & $265(26,8)$ & \\
\hline Preta & $46(8,0)$ & $187(15,7)$ & $77(11,1)$ & $177(17,9)$ & \\
\hline Amarela & $26(4,5)$ & $31(2,6)$ & $46(6,6)$ & $28(2,8)$ & \\
\hline Indígena & $1(0,2)$ & $15(1,3)$ & $4(0,6)$ & $11(1,1)$ & \\
\hline Educação (\%) & & & & & $<0,0001$ \\
\hline Menos que Ens. Médio & $34(5,9)$ & $260(21,7)$ & $44(6,2)$ & $122(12,2)$ & \\
\hline Ens. médio e superior & $145(25)$ & $551(45,9)$ & $119(16,8)$ & $450(45)$ & \\
\hline $\begin{array}{l}\text { incompleto } \\
\text { Superior completo ou mais }\end{array}$ & $402(69,2)$ & $389(32,4)$ & $545(77)$ & $429(42,9)$ & \\
\hline $\begin{array}{l}\text { Índice de massa corpórea - IMC } \\
\mathrm{kg} / \mathrm{m}^{2}\left(\mathrm{DP}^{*}\right)\end{array}$ & $27(4,6)$ & $27,2(4,6)$ & $26,7(4,6)$ & $27,4(5,2)$ & $\begin{array}{l}0,01 \\
0,006 * *\end{array}$ \\
\hline Circunferência da cintura $(\mathrm{cm})$ & $90(12,4)$ & $89,4(12)$ & $88,1(12,4)$ & $89,4(12,7)$ & $\begin{array}{l}0,03 \\
0,92\end{array}$ \\
\hline Hipertensão (\%) & $129(22,2)$ & $348(29,0)$ & $157(22,2)$ & $273(27,3)$ & 0,001 \\
\hline Diabetes $(\%)$ & $89(15,3)$ & $198(16,5)$ & $120(16,9)$ & $164(16,4)$ & 0,88 \\
\hline Dislipidemia (\%) & $340(58,5)$ & $649(54,1)$ & $410(57,9)$ & $501(50)$ & 0,002 \\
\hline \multicolumn{6}{|l|}{ Tabagismo (\%) } \\
\hline Nunca & $343(59,0)$ & $641(53,4)$ & $413(58,3)$ & $507(50,6)$ & 0,003 \\
\hline Passado & $156(26,9)$ & $370(30,8)$ & $198(28,0)$ & $301(30,1)$ & \\
\hline Atual & $82(14,1)$ & $189(15,8)$ & $97(13,7)$ & $193(19,3)$ & \\
\hline \multicolumn{6}{|l|}{ Ingestão de álcool (\%) } \\
\hline Nunca & $49(8,4)$ & $159(13,3)$ & $67(9,5)$ & $123(12,3)$ & $<0,0001$ \\
\hline Passada & $80(13,8)$ & $290(24,2)$ & $99(14,0)$ & $225(22,5)$ & \\
\hline Atual & $452(77,8)$ & $751(62,6)$ & $542(76,6)$ & $653(65,2)$ & \\
\hline Atividade física (\%) & & & & & $<0,0001$ \\
\hline Baixa & $404(71,9)$ & $956(83,5)$ & $498(72,4)$ & $798(83,5)$ & \\
\hline Moderada & $92(16,4)$ & $118(10,3)$ & $107(15,6)$ & $91(9,5)$ & \\
\hline Vigorosa & $66(11,7)$ & $71(6,2)$ & $83(12,1)$ & $67(7,0)$ & \\
\hline
\end{tabular}

*DP = desvio-padrão; **P para tendência. 
A Tabela 4 mostra os modelos de regressão logística expressos em Razão de Chances (RC) com respectivos intervalos de confianças a 95\% para cada um dos domínios do estresse no trabalho pelo modelo de Karasek. Os resultados foram apresentados sem ajuste, ajustados pelo Modelo 1 que incluiu idade, sexo, raça e educação e pelo Modelo 2 que incluiu as variáveis do Modelo 1 mais índice de massa corpórea (IMC), hipertensão, diabetes, dislipidemia, tabagismo, uso de álcool e prática de atividade física.

Mesmo nos modelos sem ajuste não foi encontrada nenhuma associação entre domínios do estresse no trabalho e CAC acima de zero ou igual ou acima de 100 Agatston. O ajuste pelas variáveis sociodemográficas e pelos fatores de risco para doença cardiovascular não mudaram os resultados apresentados sem ajuste.

A Tabela 5 mostra a associação dos domínios do estresse no trabalho pelo Modelo de Karasek para EIMC utilizando duas maneiras de avaliar a EIMC: usando a média dos valores médios da EIMC e usando a média dos valores máximos. Novamente não se encontrou nenhuma associação entre os domínios de estresse no trabalho pelo Modelo de Karasek com a EIMC calculada de duas maneiras diferentes. 
Tabela 4. Modelos de regressão logística com Razões de Chance (RC) com Intervalos de Confiança a 95\% (IC 95\%) com CAC $>0$ e $\geq 100$ de acordo com os tercis de demanda, controle e suporte social.

Categorias de Escore de cálcio - RC (IC 95\%)

$$
\mathrm{CAC}^{*}>0
$$

\begin{tabular}{|c|c|c|c|c|}
\hline \multirow[t]{4}{*}{ Demanda } & & Sem ajuste & Modelo 1 & Modelo 2 \\
\hline & Baixo & Referência & Referência & Referência \\
\hline & Médio & $1,01(0,85-1,21)$ & $1,23(1,01-1,52)$ & $1,20(0,97-1,49)$ \\
\hline & Alto & $0,85(0,69-1,05)$ & $1,16(0,90-1,48)$ & $1,17(0,90-1,51)$ \\
\hline \multirow[t]{4}{*}{ Controle } & & Sem ajuste & Modelo 1 & Modelo 2 \\
\hline & Alto & Referência & Referência & Referência \\
\hline & Médio & $0,61(0,50-0,75)$ & $0,99(0,78-1,26)$ & $1,01(0,79-1,29)$ \\
\hline & Baixo & $0,57(0,46-0,69)$ & $0,93(0,72-1,22)$ & $0,96(0,73-1,26)$ \\
\hline \multirow[t]{4}{*}{ Suporte Social } & & Sem ajuste & Modelo 1 & Modelo 2 \\
\hline & Alto & Referência & Referência & Referência \\
\hline & Médio & $0,87(0,72-1,06)$ & $1,14(0,91-1,44)$ & $1,15(0,91-1,46)$ \\
\hline & Baixo & $0,72(0,58-0,88)$ & $0,99(0,78-1,27)$ & $0,96(0,75-1,25)$ \\
\hline \multicolumn{5}{|c|}{$\mathrm{CAC}^{*} \geq 100$} \\
\hline \multirow[t]{4}{*}{ Demanda } & & Sem ajuste & Modelo 1 & Modelo 2 \\
\hline & Baixo & Referência & Referência & Referência \\
\hline & Médio & $0,93(0,70-1,24)$ & $1,19(0,87-1,63)$ & $1,18(0,85-1,64)$ \\
\hline & Alto & $0,65(0,45-0,94)$ & $0,97(0,64-1,46)$ & $1,01(0,66-1,55)$ \\
\hline \multirow[t]{4}{*}{ Controle } & & Sem ajuste & Modelo 1 & Modelo 2 \\
\hline & Alto & Referência & Referência & Referência \\
\hline & Médio & $0,54(0,39-0,74)$ & $0,89(0,61-1,29)$ & $0,84(0,57-1,23)$ \\
\hline & Baixo & $0,60(0,43-0,83)$ & $1,00(0,66-1,53)$ & $0,90(0,58-1,38)$ \\
\hline \multirow[t]{4}{*}{ Suporte social } & & Sem ajuste & Modelo 1 & Modelo 2 \\
\hline & Alto & Referência & Referência & Referência \\
\hline & Médio & $0,64(0,47-0,88)$ & $0,84(0,59-1,18)$ & $0,80(0,56-1,23)$ \\
\hline & Baixo & $0,61(0,44-0,85)$ & $0,93(0,64-1,35)$ & $0,83(0,55-1,13)$ \\
\hline
\end{tabular}

Modelo 1: Ajustado para idade, sexo, raça e educação; Modelo 2 = Modelo 1 mais índice de massa corpórea (IMC), hipertensão, diabetes, dislipidemia, tabagismo, uso de álcool e atividade física.

*CAC $=$ Escore de cálcio 
Tabela 5. Modelos de regressão logística com Razões de Chance (RC) com Intervalos de Confiança a 95\% (IC 95\%) com a EIMC (média dos valores médios) >P75\% e a EIMC (média dos valores máximos) de acordo com os tercis de demanda, controle e suporte social.

\section{EIMC* $>$ P75}

EIMC (média dos valores médios) >P75\% RC (IC95\%)

\begin{tabular}{|c|c|c|c|c|}
\hline \multirow[t]{4}{*}{ Demanda } & & Sem ajuste & Modelo 1 & Modelo 2 \\
\hline & Baixo & Referência & Referência & Referência \\
\hline & Médio & $0,96(0,81-1,15)$ & $1,12(0,92-1,36)$ & $1,07(0,87-1,31)$ \\
\hline & Alto & $0,89(0,73-1,10)$ & $1,12(0,89-1,41)$ & $1,06(0,83-1,36)$ \\
\hline \multirow[t]{4}{*}{ Controle } & & Sem ajuste & Modelo 1 & Modelo 2 \\
\hline & Alto & Referência & Referência & Referência \\
\hline & Médio & $0,66(0,54-0,80)$ & $0,89(0,71-1,11)$ & $0,89(0,70-1,13)$ \\
\hline & Baixo & $0,73(0,60-0,89)$ & $0,94(0,73-1,20)$ & $0,89(0,68-1,16)$ \\
\hline \multirow[t]{4}{*}{ Suporte Social } & & Sem ajuste & Modelo 1 & Modelo 2 \\
\hline & Alto & Referência & Referência & Referência \\
\hline & Médio & $0,81(0,66-0,98)$ & $1,03(0,83-1,29)$ & $1,03(0,82-1,30)$ \\
\hline & Baixo & $0,83(0,67-1,01)$ & $1,14(0,91-1,44)$ & $1,12(0,88-1,43)$ \\
\hline
\end{tabular}

\section{EIMC* (média dos valores máximos) >P75\%}

Demanda

\begin{tabular}{cccc}
\cline { 2 - 4 } Baixo & Sem ajuste & Modelo 1 & Modelo 2 \\
\cline { 2 - 4 } Médio & Referência & Referência & Referência \\
Alto & $1,00(0,84-1,19)$ & $1,17(0,97-1,42)$ & $1,13(0,92-1,38)$ \\
& $0,91(0,74-1,11)$ & $1,14(0,91-1,43)$ & $1,12(0,88-1,42)$ \\
\cline { 2 - 4 } & Sem ajuste & Modelo 1 & Modelo 2 \\
\cline { 2 - 4 } Alto & Referência & Referência & Referência \\
Médio & $0,70(0,57-0,85)$ & $0,89(0,71-1,11)$ & $0,87(0,69-1,12)$ \\
Baixo & $0,81(0,66-0,99)$ & $0,97(0,76-1,23)$ & $0,91(0,70-1,19)$ \\
\cline { 2 - 4 } Alto & Sem ajuste & Modelo 1 & Rodelo 2 \\
Médio & $0,83(0,68-1,01)$ & $1,05(0,85-1,30)$ & $1,05(0,87-1,41)$ \\
Baixo & $0,83(0,67-1,02)$ & $1,13(0,90-1,42)$ & $1,11(0,87-1,41)$ \\
\hline
\end{tabular}

Controle

Baixo

Médio

Alto

Alto

Médio

Baixo

Suporte Social

Modelo 1: Ajustado para idade, sexo, raça e educação; Modelo 2: Modelo 1 mais índice de massa corpórea (IMC), hipertensão, diabetes, dislipidemia, tabagismo, uso de álcool e atividade física.

*EIMC = Espessura média-íntima de carótida. 
Tabela 6. Modelos de regressão logística com Razões de Chance (RC) com Intervalos de Confiança a 95\% (IC 95\%) com CAC $>0$ e $\geq 100$ de acordo com tercis de demanda, habilidade, autoridade decisória e suporte social.

Categorias de Escore de cálcio - RC (IC 95\%)

$\mathrm{CAC}^{*}>0$

\begin{tabular}{|c|c|c|c|c|}
\hline \multirow[t]{4}{*}{ Demanda } & & Sem ajuste & Modelo 1 & Modelo 2 \\
\hline & Baixo & Referência & Referência & Referência \\
\hline & Médio & $1,01(0,85-1,21)$ & $1,23(1,01-1,52)$ & $1,20(0,97-1,49)$ \\
\hline & Alto & $0,85(0,69-1,05)$ & $1,16(0,90-1,48)$ & $1,17(0,90-1,51)$ \\
\hline Uso & & Sem ajuste & Modelo 1 & Modelo 2 \\
\hline \multirow[t]{3}{*}{ Habilidades } & Alto & Referência & Referência & Referência \\
\hline & Médio & $0,66(0,55-0,79)$ & $0,96(0,77-1,19)$ & $0,94(0,75-1,18)$ \\
\hline & Baixo & $0,71(0,58-0,88)$ & $0,99(0,76-1,28)$ & $0,99(0,63-1,30)$ \\
\hline \multirow{4}{*}{$\begin{array}{l}\text { Autoridade } \\
\text { decisória }\end{array}$} & & Sem ajuste & Modelo 1 & Modelo 2 \\
\hline & Alto & Referência & Referência & Referência \\
\hline & Médio & $0,68(0,55-0,84)$ & $0,95(0,75-1,22)$ & $0,96(0,74-1,23)$ \\
\hline & Baixo & $0,65(0,53-0,80)$ & $1,03(0,80-1,34)$ & $1,08(0,83-1,40)$ \\
\hline \multirow{4}{*}{$\begin{array}{l}\text { Suporte } \\
\text { social }\end{array}$} & & Sem ajuste & Modelo 1 & Modelo 2 \\
\hline & Alto & Referência & Referência & Referência \\
\hline & Médio & $0,87(0,72-1,06)$ & $1,14(0,91-1,44)$ & $1,15(0,91-1,46)$ \\
\hline & Baixo & $0,72(0,58-0,88)$ & $0,99(0,78-1,27)$ & $0,96(0,75-1,25)$ \\
\hline \multicolumn{5}{|c|}{$\mathrm{CAC}^{*} \geq 100$} \\
\hline \multirow[t]{4}{*}{ Demanda } & & Sem ajuste & Modelo 1 & Modelo 2 \\
\hline & Baixo & Referência & Referência & Referência \\
\hline & Médio & $0,93(0,70-1,24)$ & $1,19(0,87-1,63)$ & $1,18(0,85-1,64)$ \\
\hline & Alto & $0,65(0,45-0,94)$ & $0,97(0,64-1,46)$ & $1,01(0,67-1,56)$ \\
\hline Uso & & Sem ajuste & Modelo 1 & Modelo 2 \\
\hline \multirow[t]{3}{*}{ Habilidades } & Alto & Referência & Referência & Referência \\
\hline & Médio & $0,82(0,59-1,16)$ & $1,28(0,90-1,83)$ & $1,19(0,83-1,72)$ \\
\hline & Baixo & $0,80(0,59-1,07)$ & $1,22(0,80-1,27)$ & $1,05(0,68-1,63)$ \\
\hline \multirow{3}{*}{$\begin{array}{l}\text { Autoridade } \\
\text { decisória }\end{array}$} & & Sem ajuste & Modelo 1 & Modelo 2 \\
\hline & Alto & Referência & Referência & Referência \\
\hline & Médio & $0,75(0,53-1,05)$ & $1,17(0,80-1,71)$ & $1,14(0,77-1,68)$ \\
\hline
\end{tabular}




\begin{tabular}{lcccc}
\multirow{2}{*}{$\begin{array}{l}\text { Suporte } \\
\text { social }\end{array}$} & Baixo & $0,69(0,49-0,97)$ & $1,18(0,79-1,78)$ & $1,16(0,76-1,77)$ \\
\cline { 3 - 5 } & & Sem ajuste & Modelo 1 & Modelo 2 \\
\cline { 3 - 4 } & Alto & Referência & Referência & Referência \\
& Médio & $0,64(0,47-0,88)$ & $0,84(0,59-1,18)$ & $0,80(0,56-1,23)$ \\
& Baixo & $0,61(0,44-0,85)$ & $0,93(0,64-1,35)$ & $0,83(0,55-1,13)$
\end{tabular}

Modelo 1: Ajustado para idade, sexo, raça e educação; Modelo 2 = Modelo 1 mais índice de massa corpórea (IMC), hipertensão, diabetes, dislipidemia, tabagismo, uso de álcool e atividade física.

*CAC $=$ Escore de cálcio

A Tabela 6 mostra a mesma associação considerando-se com CAC $>0$ e $\geq 100$ de acordo com tercis de demanda, uso de demanda, uso habilidade, autoridade decisória e suporte social. Os resultados para uso de habilidades e autoridade decisória são também sem significância mesmo no modelo sem ajuste.

A Tabela 7 mostra a associação de uso de habilidades e autoridade decisória com a EIMC calculada como a média dos valores médios e como a média dos valores máximos $>\mathrm{P} 75 \%$. Novamente nenhum resultado significativo foi encontrado. 
Tabela 7. Modelos de regressão logística com Razões de Chance (RC) com Intervalos de Confiança a 95\% (IC 95\%) com a EIMC (média dos valores médios) >P75 e a EIMC (média dos valores máximos) de acordo com os tercis de demanda, habilidades, autoridade decisória e suporte social.

\section{EIMC* $>$ P75}

EIMC* (média dos valores médios) >P75 RC (IC95\%)

\begin{tabular}{|c|c|c|c|c|}
\hline \multirow[t]{4}{*}{ Demanda } & & Sem ajuste & Modelo 1 & Modelo 2 \\
\hline & Baixo & Referência & Referência & Referência \\
\hline & Médio & $0,96(0,81-1,15)$ & $1,12(0,92-1,36)$ & $1,07(0,87-1,31)$ \\
\hline & Alto & $0,89(0,73-1,10)$ & $1,12(0,89-1,41)$ & $1,06(0,83-1,36)$ \\
\hline Uso & & Sem ajuste & Modelo 1 & Modelo 2 \\
\hline \multirow[t]{3}{*}{ Habilidades } & Alto & Referência & Referência & Referência \\
\hline & Médio & $0,85(0,71-1,02)$ & $1,10(0,89-1,35)$ & $1,11(0,89-1,39)$ \\
\hline & Baixo & $0,93(0,76-1,14)$ & $1,05(0,82-1,34)$ & $1,05(0,81-1,36)$ \\
\hline \multirow{4}{*}{$\begin{array}{l}\text { Autoridade } \\
\text { decisória }\end{array}$} & & Sem ajuste & Modelo 1 & Modelo 2 \\
\hline & Alto & Referência & Referência & Referência \\
\hline & Médio & $0,73(0,59-0,89)$ & $0,94(0,75-1,18)$ & $0,91(0,72-1,16)$ \\
\hline & Baixo & $0,72(0,58-0,88)$ & $0,92(0,73-1,18)$ & $0,89(0,69-1,14)$ \\
\hline \multirow{4}{*}{$\begin{array}{l}\text { Suporte } \\
\text { social }\end{array}$} & & Sem ajuste & Modelo 1 & Modelo 2 \\
\hline & Alto & Referência & Referência & Referência \\
\hline & Médio & $0,81(0,66-0,98)$ & $1,03(0,83-1,29)$ & $1,03(0,82-1,30)$ \\
\hline & Baixo & $0,83(0,67-1,01)$ & $1,14(0,91-1,44)$ & $1,12(0,88-1,43)$ \\
\hline
\end{tabular}

EIMC* (média dos valores máximos) >P75 RC (IC95\%)

Demanda

Baixo

\begin{tabular}{ccc}
\hline Sem ajuste & Modelo 1 & Modelo 2 \\
\hline Referência & Referência & Referência \\
$1,00(0,84-1,19)$ & $1,17(0,97-1,42)$ & $1,13(0,92-1,38)$ \\
$0,91(0,74-1,11)$ & $1,14(0,91-1,43)$ & $1,12(0,88-1,42)$ \\
\hline
\end{tabular}

Uso de

Médio

Sem ajuste

Modelo 1

Modelo 2

Habilidades Alto

Referência

Referência

Referência

Médio

$$
\text { 0,87 (0,72-1,04) }
$$

$1,04(0,85-1,28)$

$1,07(0,86-1,34)$

Baixo

1,01 (0,83-1,23)

$1,07(0,85-1,36)$

1,06 (0,82-1,37)

Autoridade

decisória

Alto

\begin{tabular}{cc}
\hline Sem ajuste & Modelo 1 \\
\hline Referência & Referência
\end{tabular}

Modelo 2

Referência

Médio

$0,76(0,62-0,94)$

$0,95(0,76-1,19)$

$0,93(0,73-1,19)$ 


\begin{tabular}{lcccc}
\multirow{2}{*}{$\begin{array}{l}\text { Suporte } \\
\text { social }\end{array}$} & Baixo & $0,81(0,66-1,00)$ & $1,01(0,80-1,28)$ & $0,97(0,74-1,25)$ \\
\cline { 3 - 5 } & & Sem ajuste & Modelo 1 & Modelo 2 \\
\cline { 3 - 4 } & Alto & Referência & Referência & Referência \\
& Médio & $0,83(0,68-1,01)$ & $1,05(0,85-1,30)$ & $1,05(0,87-1,41)$ \\
& Baixo & $0,83(0,67-1,02)$ & $1,13(0,90-1,42)$ & $1,11(0,87-1,41)$ \\
\hline
\end{tabular}

Modelo 1: Ajustado para idade, sexo, raça e educação; Modelo $2=$ Modelo 1 mais índice de massa corpórea (IMC), hipertensão, diabetes, dislipidemia, tabagismo, uso de álcool e atividade física.

*EIMC = Espessura média-íntima de carótida.

As Tabelas 8, 910 e 11 mostram os modelos de regressão linear ajustados pelas mesmas variáveis usadas no modelo logístico.

A Tabela 8 mostra os modelos lineares para a associação entre os domínios do modelo de Karasek e a EIMC calculada como a média dos valores médios. Exceto pela associação de apoio social baixo e médio com valores mais baixos de EIMC medida como a média dos valores médios que foram significativos no modelo sem ajuste, não se encontrou nenhuma outra associação.

A Tabela 9 mostra a mesma análise considerando-se o uso de habilidades e a autoridade decisória para a EIMC medida como a média dos valores médios. Exceto pela associação de apoio social baixo e médio com valores mais baixos de EIMC medida como a média dos valores médios que foram significativos no modelo sem ajuste, não se encontrou nenhuma outra associação. 
Tabela 8. Beta (Intervalo de Confiança a 95\%) da associação entre tercis de demanda, controle e apoio social e espessura de íntima-média de carótidas (média dos valores médios) sem ajuste, ajustado por fatores sociodemográficos e após ajuste multivariado.

\begin{tabular}{|c|c|c|c|c|}
\hline \multirow[t]{8}{*}{ Demanda } & & Sem ajuste & Modelo 1 & Modelo 2 \\
\hline & Baixo & Referência & Referência & Referência \\
\hline & Médio & -0.004 & 0.004 & 0.002 \\
\hline & & $(-0.013$ to 0.005$)$ & $(-0.004$ to 0.012$)$ & $(-0.006$ to 0.010$)$ \\
\hline & & $\mathrm{P}=0.40$ & $\mathrm{P}=0.33$ & $P=0.61$ \\
\hline & Alto & -0.010 & 0.04 & 0.003 \\
\hline & & $(-0.021$ to 0.001$)$ & $(-0.006$ to 0.013$)$ & $(-0.007$ to 0.013$)$ \\
\hline & & $P=0.06$ & $P=0.47$ & $\mathrm{P}=0.58$ \\
\hline \multirow[t]{8}{*}{ Controle } & & Sem ajuste & Modelo 1 & Modelo 2 \\
\hline & Alto & Referência & Referência & Referência \\
\hline & Médio & -0.023 & -0.004 & -0.007 \\
\hline & & $(-0.033$ to -0.012$)$ & $(-0.014$ to 0.006 & $(-0.016$ to 0.003$)$ \\
\hline & & $\mathrm{P}<0.0001$ & $P=0.41$ & $P=0.49$ \\
\hline & Baixo & -0.015 & 0.0001 & -0.004 \\
\hline & & $(-0.026$ to -0.04$)$ & $(-0.011$ to 0.011$)$ & $(-0.014$ to 0.007$)$ \\
\hline & & $P=0.005$ & $\mathrm{P}=0.99$ & $\mathrm{P}=0.49$ \\
\hline \multirow{8}{*}{$\begin{array}{l}\text { Suporte } \\
\text { social }\end{array}$} & & Sem ajuste & Modelo 1 & Modelo 2 \\
\hline & Alto & Referência & Referência & Referência \\
\hline & Médio & -0.011 & 0.006 & 0.005 \\
\hline & & $(-0.022$ to -0.001$)$ & $(-0.004$ to 0.015$)$ & $(-0.004$ to 0.015$)$ \\
\hline & & $P=0.03$ & $P=0.22$ & $P=0.26$ \\
\hline & Baixo & -0.012 & 0.009 & 0.006 \\
\hline & & $(-0.023$ to -0.002$)$ & $(-0.001$ to 0.019$)$ & $(-0.004$ to 0.016$)$ \\
\hline & & $P=0.03$ & 0.22 & $\mathrm{P}=0.21$ \\
\hline
\end{tabular}

Modelo 1: Ajustado para idade, sexo, raça e educação; Modelo 2 = Modelo 1 mais índice de massa corpórea (IMC), hipertensão, diabetes, dislipidemia, tabagismo, uso de álcool e atividade física.

*EIMC = Espessura média-íntima de carótida. 
Tabela 9. Beta e Intervalo de Confiança a $95 \%$ da associação de tercis de demanda, uso de habilidade, autoridade decisória e apoio social com a espessura de íntima-média de carótidas (média dos valores médios) sem ajuste, ajustado por fatores sociodemográficos e ajuste multivariado.

\begin{tabular}{|c|c|c|c|c|}
\hline \multirow[t]{8}{*}{ Demanda } & & Sem ajuste & Modelo 1 & Modelo 2 \\
\hline & Baixo & Referência & Referência & Referência \\
\hline & Médio & -0.004 & 0.004 & 0.002 \\
\hline & & $(-0.013$ to 0.005$)$ & $(-0.004$ to 0.012$)$ & $(-0.006$ to 0.010$)$ \\
\hline & & $P=0.40$ & $P=0.33$ & $P=0.61$ \\
\hline & Alto & -0.010 & 0.04 & 0.003 \\
\hline & & $(-0.021$ to 0.001$)$ & $(-0.006$ to 0.013$)$ & $(-0.007$ to 0.013$)$ \\
\hline & & $P=0.06$ & $P=0.47$ & $P=0.58$ \\
\hline Uso & & Sem ajuste & Modelo 1 & Modelo 2 \\
\hline \multirow[t]{8}{*}{ Habilidades } & Alto & Referência & Referência & Referência \\
\hline & Médio & -0.013 & -0.001 & -0.001 \\
\hline & & $(-0.023$ to - & $(-0.010$ to 0.008$)$ & $(-0.012$ to 0.009$)$ \\
\hline & & $0.004)$ & $P=0.85$ & 0.81 \\
\hline & & $P=0.006$ & & \\
\hline & Baixo & -0.04 & 0.0001 & -0.002 \\
\hline & & $(-0.015$ to 0.06$)$ & $(-0.010$ to 0.010$)$ & -0.001 to 0.006$)$ \\
\hline & & $P=0.42$ & 0.99 & $P=0.60$ \\
\hline \multirow{9}{*}{$\begin{array}{l}\text { Autoridade } \\
\text { decisória }\end{array}$} & & Sem ajuste & Modelo 1 & Modelo 2 \\
\hline & Alto & Referência & Referência & Referência \\
\hline & Médio & -0.022 & -0.005 & -0.007 \\
\hline & & $(-0.033$ to - & $(-0.016$ to 0.005$)$ & $(-0.017$ to 0.003$)$ \\
\hline & & $0.011)$ & $P=0.29$ & $P=0.15$ \\
\hline & & $\mathrm{P}<0.0001$ & & \\
\hline & Baixo & -0.018 & -0.001 & $-0.004(-0.014$ to 0.007$)$ \\
\hline & & $(-0.029$ to -0.007 & $(-0.011$ to 0.009$)$ & $P=0.50$ \\
\hline & & $P=0.001$ & $P=0.84$ & \\
\hline \multirow{4}{*}{$\begin{array}{l}\text { Suporte } \\
\text { social }\end{array}$} & & Sem ajuste & Modelo 1 & Modelo 2 \\
\hline & Alto & Referência & Referência & Referência \\
\hline & Médio & -0.011 & 0.006 & 0.005 \\
\hline & & & $(-0.004$ to 0.015$)$ & $(-0.004$ to 0.015$)$ \\
\hline
\end{tabular}




$\begin{array}{ccc}(-0.022 \text { to }- & P=0.22 & P=0.26 \\ 0.001) & \\ P=0.03 & \end{array}$

\begin{tabular}{|c|c|c|c|}
\hline \multirow[t]{3}{*}{ Baixo } & -0.012 & 0.009 & 0.006 \\
\hline & $(-0.023$ to 0.002$)$ & $(-0.001$ to 0.019$)$ & $(-0.004$ to 0.016$)$ \\
\hline & $P=0.03$ & 0.22 & $\mathrm{P}=0.21$ \\
\hline
\end{tabular}

Modelo 1: Ajustado para idade, sexo, raça e educação; Modelo $2=$ Modelo 1 mais índice de massa corpórea (IMC), hipertensão, diabetes, dislipidemia, tabagismo, uso de álcool e atividade física.

*EIMC = Espessura média-íntima de carótida.

A Tabela 10 mostra os modelos lineares para a associação entre os domínios do modelo de Karasek e a EIMC calculada como a média dos valores máximos. Exceto pela associação borderline de controle baixo e EIMC no modelo sem ajuste de controle baixo com a EIMC medida como a média dos valores máximos que foram significativos no modelo sem ajuste, que foram significativos no modelo sem ajuste, não se encontrou nenhuma outra associação.

A Tabela 11 mostra os modelos lineares para a associação entre os domínios uso de habilidades e autoridade decisória e a EIMC calculada como a média dos valores máximos. Exceto pela associação uso de habilidades baixo e EIMC no modelo sem ajuste que perdeu significância após o ajuste por fatores sociodemográficos, nenhuma outra associação se mostrou significativa. 
Tabela 10. Beta (Intervalo de Confiança a 95\%) da associação entre tercis de demanda, controle e apoio social e Espessura de íntima-média de carótidas (média dos valores máximos) sem ajuste, ajustado por fatores sociodemográficos e após ajuste multivariado.

\begin{tabular}{|c|c|c|c|c|}
\hline \multirow[t]{8}{*}{ Demanda } & & Sem ajuste & Modelo 1 & Modelo 2 \\
\hline & Baixo & Referência & Referência & Referência \\
\hline & Médio & -0.001 & 0.009 & 0.007 \\
\hline & & $(-0.013$ to 0.010$)$ & $(-0.001$ to 0.02$)$ & $(-0.004$ to 0.017$)$ \\
\hline & & $P=0.82$ & $P=0.09$ & $P=0.20$ \\
\hline & Alto & -0.010 & 0.008 & 0.008 \\
\hline & & $(-0.023$ to 0.004$)$ & $(-0.04$ to 0.021$)$ & $(-0.005$ to 0.02$)$ \\
\hline & & $\mathrm{P}=0.17$ & $\mathrm{P}=0.19$ & $\mathrm{P}=0.24$ \\
\hline \multirow[t]{8}{*}{ Controle } & & Sem ajuste & Modelo 1 & Modelo 2 \\
\hline & Alto & Referência & Referência & Referência \\
\hline & Médio & 0.024 & -0.003 & -0.007 \\
\hline & & $(-0.037$ to -0.010$)$ & $(-0.015$ to 0.010 & $(-0.019$ to 0.006$)$ \\
\hline & & $P=0.001$ & $P=0.67$ & $P=0.29$ \\
\hline & Baixo & -0.014 & 0.001 & -0.004 \\
\hline & & $(-0.028$ to 0.0001 & $(-0.013$ to 0.015$)$ & $(-0.018$ to 0.009$)$ \\
\hline & & $\mathrm{P}=0.05$ & $P=0.89$ & $P=0.52$ \\
\hline \multirow{8}{*}{$\begin{array}{l}\text { Suporte } \\
\text { social }\end{array}$} & & Sem ajuste & Modelo 1 & Modelo 2 \\
\hline & Alto & Referência & Referência & Referência \\
\hline & Médio & -0.018 & 0.003 & 0.003 \\
\hline & & $(-0.031$ to -0.004$)$ & $(-0.009$ to 0.016$)$ & $(-0.009$ to 0.015$)$ \\
\hline & & $\mathrm{P}=0.01$ & $P=0.57$ & $P=0.50$ \\
\hline & Baixo & -0.017 & 0.010 & 0.007 \\
\hline & & $(-0.030$ to -0.003$)$ & $(-0.003$ to 0.023$)$ & $(-0.006$ to 0.020$)$ \\
\hline & & $P=0.02$ & $P=0.13$ & $P=0.27$ \\
\hline
\end{tabular}

Modelo 1: Ajustado para idade, sexo, raça e educação; Modelo 2 = Modelo 1 mais índice de massa corpórea (IMC), hipertensão, diabetes, dislipidemia, tabagismo, uso de álcool e atividade física.

*EIMC = Espessura média-íntima de carótida. 
Tabela 11. Beta e Intervalo de Confiança a 95\% da associação de tercis de demanda, uso de habilidade, autoridade decisória e apoio social com a espessura de íntima-média de carótidas (média dos valores máximos) sem ajuste, ajustado por fatores sociodemográficos e ajuste multivariado.

\begin{tabular}{|c|c|c|c|c|}
\hline \multirow[t]{8}{*}{ Demanda } & & Sem ajuste & Modelo 1 & Modelo 2 \\
\hline & Baixo & Reference & Reference & Reference \\
\hline & Médio & -0.001 & 0.005 & 0.007 \\
\hline & & $(-0.013$ to 0.010$)$ & $(-0.001$ to 0.02$)$ & $(-0.004$ to 0.017$)$ \\
\hline & & $P=0.82$ & $\mathrm{P}=0.009$ & $\mathrm{P}=0.20$ \\
\hline & Alto & -0.010 & 0.008 & 0.008 \\
\hline & & $(-0.023$ to 0.004$)$ & $(-0.04$ to 0.021$)$ & $(-0.005$ to 0.02$)$ \\
\hline & & $\mathrm{P}=0.17$ & $P=0.19$ & $\mathrm{P}=0.24$ \\
\hline Uso & & Sem ajuste & Modelo 1 & Modelo 2 \\
\hline \multirow[t]{7}{*}{ habilidades } & Alto & Reference & Reference & Reference \\
\hline & Médio & -0.012 & 0.001 & -0.001 \\
\hline & & $(-0.024$ to 0.0001$)$ & $(-0.010$ to 0.012$)$ & $(-0.012$ to 0.010$)$ \\
\hline & & $P=0.053$ & $P=0.86$ & P 0.83 \\
\hline & Baixo & 0.0001 & 0.003 & -0.01 \\
\hline & & $(-0.013$ to 0.014$)$ & $(-0.010$ to 0.016$)$ & $(-0.014$ to 0.013$)$ \\
\hline & & $P=0.95$ & $P=0.67$ & $P=0.93$ \\
\hline Autoridade & & Sem ajuste & Modelo 1 & Modelo 2 \\
\hline \multirow[t]{7}{*}{ Decisória } & Alto & Reference & Reference & Reference \\
\hline & Médio & -0.025 & -0.006 & -0.010 \\
\hline & & $(-0.039$ to -0.011$)$ & $(-0.019$ to 0.008$)$ & $(-0.022$ to 0.003$)$ \\
\hline & & $P=0.001$ & $P=0.41$ & $P=0.14$ \\
\hline & Baixo & -0.017 & 0.001 & -0.004 \\
\hline & & $(-0.031$ to -.003$)$ & $(-0.012$ to 0.015$)$ & $(-0.017$ to 0.010$)$ \\
\hline & & & $P=0.86$ & $P=0.60$ \\
\hline Suporte & & Sem ajuste & Modelo 1 & Modelo 2 \\
\hline \multirow[t]{5}{*}{ Social } & Alto & Reference & Reference & Reference \\
\hline & Médio & -0.018 & 0.003 & 0.003 \\
\hline & & $(-0.031$ to -0.004$)$ & $(-0.009$ to 0.016$)$ & $(-0.009$ to 0.015$)$ \\
\hline & & $P=0.01$ & $P=0.57$ & $P=0.50$ \\
\hline & Baixo & -0.017 & 0.010 & 0.007 \\
\hline
\end{tabular}




$$
\begin{array}{ccc}
(-0.030 \text { to }-0.003) & (-0.003 \text { to } 0.023) & (-0.006 \text { to } 0.020) \\
\mathrm{P}=0.02 & \mathrm{P}=0.13 & \mathrm{P}=0.27
\end{array}
$$

Modelo 1: Ajustado para idade, sexo, raça e educação; Modelo $2=$ Modelo 1 mais índice de massa corpórea (IMC), hipertensão, diabetes, dislipidemia, tabagismo, uso de álcool e atividade física.

*EIMC = Espessura média-íntima de carótida. 


\section{Discussão}

Houve uma ausência de associação entre os domínios de estresse no trabalho e CAC utilizando como medida de aterosclerose subclínica o CAC $>0$ e $C A C \geq 100$ unidades Agatston. Também não foi observada associação entre estresse no trabalho e a EIMC $>$ P75\%, tanto para o EIMC calculada como a média dos valores médios, como a calculada como a média dos valores máximos. Houve uma associação no modelo sem ajustes de baixo nível de apoio com a EIMC como variável contínua usando a média das médias para cálculo da EIMC no modelo sem ajustes, mas perdeu a significância após ajuste pelas variáveis sociodemográficas. A medida da EIMC como média dos valores máximos mostrou uma associação entre controle e EIMC usando o modelo clássico de Karasek, mas também houve perda da significância após ajuste pelas variáveis sociodemográficas. Utilizando-se a versão modificada do modelo de Karasek, houve uma associação entre menor uso de habilidades com a EIMC no modelo sem ajustes, porém este perdeu a significância após ajuste por variáveis sociodemográficas.

\subsection{Estresse no trabalho e espessura íntima-média de carótidas}

Vários estudos avaliaram de forma transversal a associação de estresse no trabalho e aterosclerose subclínica mensurada pela EIMC (Muntaner et al., 1998; Kamarck et al., 2004; Rosvall et al., 2002; Nordstrom et al., 2001; Jedryka-Goral et al., 2006). Em uma amostra de 10.801 participantes com 45 a 64 anos, o Atherosclerosis Risk in Communities Study (ARIC) avaliou o controle sobre o trabalho nas dimensões uso de habilidades e autoridade decisória. Embora não tenha sido aplicada nenhuma escala específica para 
avaliar o uso de habilidades e a autoridade decisória, essas informações foram derivadas a partir da categoria ocupacional do participante. Os autores observaram que o uso de habilidades e a autoridade decisória se associaram negativamente com a EIMC medida na artéria carótida comum, na bifurcação da carótida comum e na artéria carótida interna, chegando à conclusão que após ajuste multivariado a associação enfraqueceu, mas persistiu significativa sugerindo que ausência de controle tem um papel na gênese da aterosclerose subclínica no estudo. (Muntaner et al., 1998)

No Pittsburgh Healthy Heart Project, 337 participantes com idades entre 50 e 70 anos responderam a um questionário que incorporou duas questões do Karasek Job Control Questionnaire para estudar a demanda e autoridade decisória. Kamarck et al. analisaram a EIMC em artéria carótida comum proximal e distal, bulbo carotídeo e artéria carótida interna distal, e realizaram uma média desses valores. Participantes que relataram demandas muito altas durante sua vida diária apresentaram valores mais elevados de EIMC mesmo após ajuste multivariado por variáveis sociodemográficas. Não se encontrou nenhuma associação com autoridade decisória. Entretanto, essa associação positiva entre demanda e EIMC foi modesta, representando somente $2 \%$ da variância da EIMC (Kamarck et al., 2004).

Rosvall et al., utilizando-se de amostra da coorte Malmo Diet and Cancer Study, realizaram estudo transversal aninhado com 2658 participantes com idades entre 46 e 65 anos sem antecedente de doença cardiovascular prévia, e avaliaram a associação entre demanda e autoridade decisória no ambiente de trabalho, baseados no questionário de Karasek, com EIMC, avaliado tanto em artéria carótida comum quanto em bifurcação carotídea, e placas em carótidas. Participantes mulheres com altas demandas e baixa autoridade decisória no trabalho apresentaram maiores valores de EIMC em bifurcação carotídea e maior incidência de placas carotídeas (definidas como EIMC >1,2mm), porém 
tal perfil também fora observado em participantes mulheres com altas demandas psicológicas e alta autoridade decisória (trabalhos ativos conforme Karasek). Nenhuma associação nos valores de EIMC com a variáveis de Karasek fora observada em homens (Rosvall et al., 2002).

Comparadas a essas três análises, nossos resultados não mostraram nenhuma associação. Essas diferenças poderiam ser explicadas em parte pela forma de medir o estresse no trabalho entre esses três estudos e a presente análise. Embora no estudo de Kamarck et al. tenham sido usadas duas questões do questionário de Karasek, os autores não utilizaram o questionário completo. Rosvall et al. também se basearam no questionário de Karasek, porém não avaliaram o uso de habilidades ou o suporte social, enquanto no estudo com dados do ARIC Study, o estresse no trabalho tenha sido mensurado baseado nas atividades ocupacionais do participante sem a aplicação de uma escala (Muntaner et al., 1998; Rosvall et al., 2002). A amostra utilizada nesta análise é mais jovem que a amostra do ARIC (Muntaner et al., 1998), do Malmo Diet and Cancer Study (Rosvall et al., 2002) e do Pittsburgh Healthy Heart Project (Kamarck et al., 2004). Além disso, a amostra do ELSA-Brasil incluiu somente servidores públicos com nível educacional e renda mais elevadas em relação a população geral brasileira, também diferente da amostra de base comunitária do ARIC, do Malmo Diet and Cancer Study e do estudo em Pittsburgh.

Nordstrom et al. também avaliaram transversalmente estresse no trabalho e aterosclerose subclínica utilizando-se da EIMC (Nordstrom et al., 2001). Incluindo 573 funcionários de uma empresa de utilitários com idades entre 40 e 60 anos e assintomáticos para doença cardiovascular, os autores avaliaram estresse no trabalho por meio de questionário próprio, incluindo 3 questões semelhantes ao proposto por Karasek para acessar a demanda relacionada ao trabalho e outras 3 questões semelhantes ao proposto 
por Siegrist para análise das características intrínsecas ao trabalhador. Os autores observaram associação entre EIMC acima do percentil 90, aferida no segmento distal das artérias carótidas comuns, com estresse laboral no subgrupo dos homens. Em análise ajustada para a idade, a prevalência de lesões carotídeas entre homens no grupo de maior quintil para estresse laboral foi de $36 \%$, comparado com $21 \%$ no grupo de homens no menor quintil. Nenhuma associação fora observada entre as mulheres (Nordstrom et al., 2001).

Também em um estudo transversal, Jedryka-Góral et al. analisaram 150 trabalhadores de escritório com idades entre 35 e 65 anos, e avaliaram a associação de EIMC com diversos potenciais moderadores da aterosclerose tanto de caráter autoimune quanto ocupacional, incluindo um questionário de estresse relacionado ao trabalho. (Jedryka-Goral et al., 2006) Os autores utilizaram-se de uma versão adaptada ao polonês de um questionário desenvolvido por Cooper et al., avaliando o que denominaram de 'estresse global' e seus componentes: estressores particulares, sobrecarga de trabalho, relações interpessoais, desequilíbrio trabalho-casa, papel de liderança, grau de responsabilidade, preocupações diárias, condições organizacionais e técnicas de enfrentamento (Cooper et al., 2001 apud Jedryka-Goral et al., 2006) . Avaliando separadamente o grupo com presença de placa em carótida, considerado como espessamento intimal maior do que 50\% em relação à área circundante, Jedryka-Goral et al. observaram associação entre presença de placa e um dos componentes do estresse global, o desequilíbrio casa-trabalho. Não fora encontrada associação entre aumentos do EIMC e estresse global ou seus outros domínios. (Jedryka-Goral et al., 2006) 
Tanto o estudo de Nordstrom et al. quanto o de Jedryka-Goral et al. utilizaram formas de aferição de estresse laboral divergentes do utilizado no presente estudo, dificultando uma comparação acurada dos resultados. A forma de aferição da EIMC realizada por Nordstrom et al. são equivalentes ao do presente estudo, porém JedrykaGoral et al. não disponibilizou tal informação em seu estudo (Nordstrom et al. 2001; Jedryka-Goral et al. 2006).

Embora a EIMC seja preditora de doença cardiovascular (Rosvall et. al. 2005), há diferentes locais para a mensuração da medida. As diretrizes internacionais recomendam a mensuração na artéria carótida comum a 1 centímetro de distância do bulbo carotídeo, por ser a área de melhor reprodutibilidade e maior facilidade de obtenção de imagens (Mill et al., 2013). O presente estudo segue estas recomendações, também adotadas pela maior parte da literatura científica na atualidade. No entanto, há aferições na bifurcação carotídea, bulbo a e na artéria carótida interna em alguns estudos, devido ao fato da doença aterosclerótica poder ocorrer mais precocemente nestas áreas. Os autores que defendem estas localizações se baseiam na premissa de que haja acometimento inicial do processo aterosclerótico nesses locais e que as medidas nas artérias carótidas comuns podem subestimar a carga aterosclerótica global do indivíduo (Mookadam et. al. 2010). Desta maneira, cabe ressaltar a importância da descrição clara da localização da obtenção das imagens no exame de EIMC na avaliação dos estudos da área. A diversidade das formas de aferição e da topografia vascular em que são realizadas as medidas da EIMC dificultam as comparações entre os estudos, e podem ser responsáveis pelos resultados heterogêneos encontrados. Dos estudos citados anteriormente, apenas Nordstrom et al. utilizaram-se da metodologia equivalente ao do presente estudo (Nordstrom et al., 2001).

Vale a pena recordar que o objetivo da aferição de EIMC seja avaliar a espessura da parede vascular, em específico de sua camada íntima e média, pois os mecanismos 
moleculares que se traduzem em sua espessura são correlatos aos mecanismos fisiopatológicos para a formação da placa aterosclerótica. O achado de placas durante este exame já confere o risco mais elevado de DCV e se trata de avaliação à parte da EIMC.

Diferenças individuais na resposta inata ao estresse também podem justificar parte da heterogeneidade nos resultados de literatura científica relacionados ao estresse no trabalho e doença aterosclerótica. A atividade dopaminérgica parece exercer um papel central na modulação fisiológica individual ao estresse, inibindo em sistema nervoso central o tônus simpático sob o sistema cardiovascular, contra regulando a atividade do eixo hipotalâmico-pituitário-adrenal e podendo moderar os efeitos do estresse sob o risco cardiovascular. (Hintsanen et al., 2008) Com base nessa observação, Hintsanen et al. avaliaram a presença de alterações no gene COMT (Catechol-O-Methyl-Tranferase), responsável pela degradação de catecolaminas e seus neurotransmissores como a dopamina, a adrenalina e a noradrenalina, em associação com análises de EIMC e de estresse no trabalho. Segundo os autores, alterações polimórficas no gene COMT com um nucleotídeo valina conferem menores taxas de dopamina circulante, associadas a menores habilidades cognitivas para lidar com situações estressantes, gerando uma maior sensação subjetiva de estresse e sobrecarga, enquanto apresentações com o nucleotídeo metionina tornam a degradação da dopamina menos eficaz, e portanto conferem maior adaptabilidade cognitiva à situações estressantes. (Hintsanen et al., 2008)

Em estudo de corte transversal aninhado em coorte (participantes do estudo Cardiovascular Risk in Young Finns), coletado em seu $21^{\circ}$ ano de seguimento, Hintsanen et al. avaliaram 700 indivíduos finlandeses entre 24 e 39 anos, e aferiram a EIMC, a presença do gene COMT e seus polimorfismos e o estresse no trabalho por meio de questionário baseado no modelo de Karasek. Os autores observaram que o grupo de 
indivíduo com alto desgaste e com EIMC acima do terceiro tercil eram significantemente associados ao genótipo COMT com o nucleotídeo valina, conforme teorizado, porém tal associação ocorreu apenas no sexo masculino (Beta=-0,320, $\mathrm{p}=0,02)$. A associação permaneceu significativa mesmo após ajuste para idade e demais fatores de risco cardiovasculares. (Hintsanen et al., 2008). Tais achados sugerem que há outras variáveis atuando na percepção subjetiva de estresse pelo indivíduo.

Três estudos avaliaram prospectivamente a associação de estresse no trabalho com a EIMC (Rosenstrom et al., 2011; Kamarck et al., 2007/2012; Fujihiro et al., 2015). Fujishiro et al. avaliaram nos dados da coorte Multiethnic Study of Atherosclerosis (MESA) a associação das características ocupacionais com a EIMC. Incluindo 3109 participantes com idades entre 45 e 84 anos e livres de doença cardiovascular clinicamente expressa, os autores avaliaram estresse no trabalho e as dimensões demanda psicológicas laborais e controle sobre o trabalho por meio de questionário próprio, e analisaram a EIMC com mesma metodologia do presente estudo, em artéria carótida comum, com seguimento médio de 9,4 anos. Nenhuma associação fora encontrada, assim como o presente estudo (Fujishiro et al., 2015).

No The Cardiovascular Risk in Young Finns Study, foram avaliados 709 indivíduos em dois seguimentos: 2001, com idades entre 24 e 39 anos, e em 2007, quando os participantes apresentavam idades entre 30 e 45 anos. A avaliação de demanda no trabalho fora realizada por meio de questionário próprio desenvolvido e validado pelo Instituto Finlandês de Saúde Ocupacional, e o controle sobre o trabalho fora mensurado com base no questionário de Karasek. Medidas de EIMC foram realizadas em artéria carótida comum, concordante com o presente estudo. Os autores relataram ausência de associação em avaliação transversal e de forma prospectiva nas mulheres. Entretanto, em 
homens com declínio importante do estresse no trabalho de 2001 a 2007, observou-se uma progressão mais lenta da EIMC (Rosenström et al., 2011).

Análises prospectivas do Pittsburgh Healthy and Heart Project mostraram que a alta demanda e o baixo controle se associaram a progressão da EIMC nos homens, mas não nas mulheres. (Kamarck et al., 2007; Kamarck et al., 2012)

\subsection{Estresse no trabalho e escore de cálcio em artérias coronárias}

Apenas um único estudo avaliou a associação de estresse no trabalho com CAC. No estudo the Coronary Artery Risk Development in Young Adults (CARDIA), foram inclusos 2943 indivíduos com idades entre 18 e 30 anos na análise inicial. O CAC foi medido em 2000/1 e 2005/6. As características do trabalho foram mensuradas pelo modelo demanda-controle incluindo demandas psicológicas e autoridade decisória em 1987/88 e 1995/96. Os resultados mostraram que a autoridade decisória e as altas demandas psicológicas e estresse no trabalho ou em exame realizado previamente ou entre 1987/88 e 1995/96 não se associaram com a presença de CAC. Entretanto, participantes cujo trabalho foi classificado como gerencial ou professional em 1995/96 apresentaram uma probabilidade menor de CAC positivo que naqueles trabalhadores que tinham ocupações laborais. (Greenlund et al., 2010) Os resultados desta análise não associaram a estresse no trabalho a presença de CAC sendo similares aos resultados do nosso estudo. Os participantes no estudo CARDIA são mais jovens do que no ELSABrasil, fator que pode ter contribuído parcialmente para os resultados negativos em ambos os estudos. 
Somente um outro estudo no ELSA-Brasil avaliou a associação entre estresse no trabalho e aterosclerose subclínica no Brasil. Camelo et al. observaram a associação entre estresse no trabalho, EIMC e posição socioeconômica atual e pregressa. Em homens, segundo o modelo de Karasek, o trabalho de alto desgaste se associou com maior EIMC ( $\beta$ 0,028; IC 95\% 0,01-0,045). Em mulheres tanto o trabalho passivo ( $\beta 0,022$; IC 95\% 0,011-0,033) quanto o alto desgaste ( $\beta 0,014$ IC 95\% 0,001-0,026) se associou com maior EIMC. Foi ainda observado que o acúmulo de desvantagens socioeconômicas se associa tanto em homens quanto em mulheres com o EIMC. O EIMC se manteve associado aos indicadores de posição socioeconômica. Após o ajuste para estresse no trabalho e nas mulheres, o trabalho passivo se manteve associado com EIMC (significância estatística limítrofe) (Camelo et al., 2015). Este estudo levanta a questão se o estresse no trabalho é um fator de risco em si ou se trata de marcador de desvantagem socioeconômica, atuando como variável intermediária no processo de determinação social da doença cardiovascular. O papel da desvantagem socioeconômica, ainda com mecanismos desconhecidos na fisiopatologia da aterosclerose e do estresse no ambiente de trabalho, pode justificar parcialmente os resultados negativos do presente estudo, por tratar-se de amostra mais jovem e de melhor condição socioeconômica em relação à população brasileira.

É bastante provável ainda que CAC e EIMC meçam fases diferentes da aterosclerose subclínica. O CAC pode ser uma medida mais tardia do processo aterosclerótico comparada a EIMC que reflete não somente um espessamento patológico da íntima, mas também a hipertrofia da camada média. (Kathiresan et al., 2006; Touboul et al., 2012). Entretanto, usando os dois métodos para mensurar a aterosclerose subclínica, EIMC e o CAC, em nenhum se observou a presença de uma associação, seja ela mais precoce ou mais tardia. Na revisão de Wilson et al., uma das limitações era o 
fato de os autores terem identificado um viés de publicação relatando que muitos estudos com resultados positivos foram identificados, mas quase nenhum com resultados negativos. Os resultados do ELSA-Brasil se encaixariam nessa lacuna.

\subsection{Limitações e fortalezas do estudo}

Entre as limitações da análise está sua natureza transversal que permite e avaliação de associação, mas não de causalidade. O ELSA-Brasil é uma coorte de servidores públicos com nível educacional e renda mais elevados comparados a estudos prévios que relataram associação entre estresse no trabalho e aterosclerose subclínica. Além disso, a amostra do ELSA-Brasil é mais jovem do que a de outros estudos a exceção do estudo CARDIA que também não encontrou nenhuma associação. A média de idade baixa dos participantes do estudo também justifica a presença de uma prevalência baixa de aterosclerose subclínica que também pode ter contribuído para os resultados que mostraram ausência de associação entre estresse no trabalho e aterosclerose subclínica mensurada de duas maneiras diferentes.

Por outro lado, há que se destacar pontos importantes do estudo. As entrevistas foram aplicadas por entrevistadores seguindo um protocolo rígido e com controle de qualidade estrito. A medida da EIMC foi feita por software que padroniza a mensuração. Todo o treinamento para realização das medidas e aplicação dos questionários foi centralizado, garantindo a homogeneidade dos dados coletados. Além disso, o ELSABrasil traz novas informações sobre estresse no trabalho e aterosclerose subclínica em uma amostra diferente dos estudos americanos e europeus. 


\section{Conclusões}

Não houve associação entre estresse no trabalho (avaliado pelo modelo de Karasek) e medidas de aterosclerose subclínica mensuradas pelo Escore de Cálcio de artérias coronárias e pela medida da Espessura Íntima-Média de Carótidas.

A análise utilizando a apresentação clássica de estresse no trabalho em demanda, controle, e apoio social não apresentou associação positiva. A categorização do controle em uso de habilidades e autoridade decisória também não alterou os resultados que mostraram ausência de associação independente da forma de medida do estresse no trabalho.

A categorização da EIMC acima do percentil $75 \%$ ou como variável contínua apresentaram resultados semelhantes com ausência de associação.

Para o CAC, o uso dos pontos de corte diferenciados como CAC $>0$ ou CAC $\geq$ 100 não modificaram os resultados, que persistiram negativos. 


\section{Referências}

Agatston AS, Janowitz WR, Hildner FJ, et al. Quantification of coronary artery calcium using ultrafast computed tomography. Carotid intima-media thickness value distributions in the Brazilian Longitudinal Study of Adult Health (ELSA-Brasil). J Am Col Cardiol. 1990; 15: 827-32.

Aguiar OB, Fonseca MJM, Valente JG. Confiabilidade (teste-reteste) da escala sueca do Questionário Demanda-Controle entre trabalhadores de restaurantes industriais do Estado do Rio de Janeiro. Rev Bras Epidemiol. 2010;13(2):212-22.

Alves MGM, Chor D, Faerstein E, Lopes CS, Werneck GL. Versão Resumida da “Job Stress Scale": adaptação para o português. Rev Saude Publica. 2004; 38: 164-171.

Alves MGM, Hokerberg YHM, Faerstein E. Tendências e diversidades na utilização empírica do Modelo Demanda-Controle de Karasek (estresse no trabalho): Uma revisão sistemática. Rev Bras Epidemiol. 2013; 16(1):125-36.

Aquino EML, Araujo MJ, Almeida MC, et al. Participants recruitment in ELSABrasil (Brazilian Longitudinal Study for Adult Health)]. Rev Saude Publica. 2013; 47 Suppl 2: 10-18.

Aquino EML, Barreto SM, Benseñor IM, et al. Brazilian Longitudinal Study of Adult Health (ELSA-Brasil): Objectives and Design. Am J Epidemiol 2012; 175: 315-324. 
Araújo TM, Karasek R. Validity and reliability of the job content questionnaire in formal and informal jobs in Brazil. SJWEH. 2008;(6):52-59.

Asai K, Kudej RK, Shen YT, Yang GP, Takagi G, Kudej AB, et al. Peripheral vascular endothelial dysfunction and apoptosis in old monkeys. Arterioscler Thromb Vasc Biol. 2000; 20:1493-1499.

Backé EM, Seidler A, Latza U, et al. The role of psychosocial stress at work for the development of cardiovascular diseases: A systematic review. Int Arch Occup Environ Health. 2012, 85:67-79.

Benseñor IM, Griep RH, Pinto KA, et al. Routines of organization of clinical tests and interviews in the ELSA-Brasil investigation center. Rev Saude Publica. 2013; 47 (2): $37-$ 47.

Berry JD, et al. Prevalence and progression of subclinical atherosclerosis in younger adults with low short-term but high life-time estimated risk for cardiovascular disease: The coronary artery risk development in young adults study and multi-ethnic study of atherosclerosis. Circulation. 2009; 119(3), 382-389.

Bots ML, Hofman A, Grobbee DE. Increased common carotid intima-media thickness: adaptive response or a reflection of atherosclerosis? Findings from the Rotterdam study. Stroke. $1997 ; 28: 2442-7$. 
Brant LCC, Nascimento BR, Passos VMA, et al. Variations and particularities in cardiovascular disease mortality in Brazil and Brazilian states in 1990 and 2015: Estimates from the Global Burden of Disease. Rev Bras Epidemiol. 2017; 20(Suppl 01): 116-128.

Brown TM, Fee E. Walter Bradford Cannon: Pioneer physiologist of human emotions. Am J Public Health. 2002; 92 (10):1594-1595.

Camelo LV, Giatti L, Chor D, Griep RH, Benseñor IM, Santos IS, Kawachi I, Barreto SM. Associations of life course socioeconomic position and job stress with carotid intima-media thickness. The Brazilian Longitudinal Study of Adult Health (ELSABrasil). Soc Science \& Medicine. 2015; 141:91-99.

Chambless LE, Heiss G, Folsom AR, et al. Association of coronary heart disease incidence with carotid arterial wall thickness and major risk factor: The Atherosclerosis Risk in Communities (ARIC) study 1987-1993. Am J Epidemiol. 1997; 146: 483-94.

Chungkham HS, Ingre M, Karasek R, Westerlund H, Theorell T. Factor structure and longitudinal measurement invariance of the demand control support model: An evidence from the Swedish Longitudinal Occupational Survey of Health (SLOSH). PLoS One. 2013;8: e70541. 
Conroy RM, Pyorala K, Fitzgerald AP, et al. Estimation of ten-year risk of fatal cardiovascular disease in Europe: the SCORE project. Eur Heart J. 2003; 24: 987-1003.

Cooper et. al. Stress at work questionnaire. In: Widerszal-Bazyl M. Stress at Work. Polish Adaptation of Occupational Stress Indicator (OSI-2). Warszawa: CIOP, 2001 (in Polish) apud Jedryka-Goral et. al. 2006.

D’Agostino RB, Vasan RS, Pencina MJ, et al. General cardiovascular risk profile for use in primary care: the Framingham heart study. Circulation. 2008; 117: 743-753.

Detrano R, Guerci AD, Carr JJ, et al. Coronary calcium as a predictor of coronary events in four racial or ethnic groups. The Multi-Ethnic Study of Atherosclerosis (MESA). $N$ Engl J Med 2008. 358:1336-1345.

Elias-Smale SE, Proença RV, Koller MT, et al. Coronary Calcium Score Improves Classification of Coronary Heart Disease Risk in the Elderly. The Rotterdam Study. JACC 2010; 56: 1407-1414.

Eller NH, Netterstrom B. Psychosocial factors at home and at work and four-year progression in intima media thickness. Int J Behav Med. 2007; 14: 21-29.

Everson SA, Lynch JW, Chesney MA, Kaplan GA, Goldberg DE, Shade SB, et al. Interaction of workplace demands and cardiovascular reactivity in progression of carotid atherosclerosis: population-based study. BMJ. 1997;314(7080):553-558. 
Fedeli LG, Vidigal PG, Leite CM, Castilhos CD, Pimentel RA, Maniero VC, Mill JG, Lotufo PA, Pereira AC, Benseñor IM. Logistics of collection and transportation of biological samples and the organization of the central laboratory in the ELSA-Brasil. Rev Saude Publica. 2013 Jun;47 Suppl 2:63-71.

Fernández-Friera L, Ibáñez B, Fuster V. Imaging subclinical atherosclerosis: Is it ready for prime time? Review. Journal of Cardiovasc Trans Res. 2014; 7:623-634.

Folsom AR, Kronmal AR, Detrano RC, O’Leary DH, Bild DE, et al. Coronary Artery Calcification compared with Carotid Intima-Media Thickness in the prediction of cardiovascular disease incidence. The Multi-Ethnic Study of Atherosclerosis (MESA). Arch Intern Med. 2008; 168:1333-1339.

Fujishiro K, Roux AVD, Landsbergis P, Kaufman JD, Korcarz CE, Stein JH. Occupational characteristics and the progression of carotid artery intima-media thickness and plaque over 9 years: the Multi-Ethnic Study of Atheriosclerosis (MESA). Occup Environ Med. 2015;72:690-698.

Fujishiro K, Diez Roux AV, Landsbergis P, Baron S, Barr RG, Kaufman JD, et al. Associations of occupation, job control and job demands with intima-media thickness: The Multi-Ethnic Study of Atherosclerosis (MESA). Occup Environ Med. 2011; 68:319326. 
GBD 2017 Causes of Death Collaborators. Global, regional, and national age-sexspecific mortality for 282 causes of death in 195 countries and territories, 1980-2017: a systematic analysis for the Global Burden of Disease Study 2017. Lancet. 2018 Nov 10;392(10159):1736-1788. Epub 2018 Nov 8. Erratum in: Lancet. 2019 Jun 22;393(10190): e44. Lancet. 2018 Nov 17;392 (10160):2170.

GBD 2017 Mortality Collaborators. Global, regional, and national age-sex-specific mortality and life expectancy, 1950-2017: a systematic analysis for the Global Burden of Disease Study 2017. Lancet. 2018 Nov 10;392(10159):1684-1735. Epub 2018 Nov 8. Erratum in: Lancet. 2019 Jun 22;393(10190): e44.

Ginty AT, Kraynak TE, Fisher JP, Gianaros PJ. Cardiovascular and autonomic reactivity ti psychological stress: Neurophysiological substrates and links to cardiovascular disease. Auton Neurosci. 2017; 207:2-9.

Greenland P, Smith SC Jr, Grundy SM. Improving coronary heart disease risk assessment in asymptomatic people: role of traditional risk factors and noninvasive cardiovascular tests. Circulation. 2001; 104:1863-1867.

Greenlund KJ, Kiefe CI, Giles WH, Liu AK. Associations of job strain and occupation with subclinical atherosclerosis: The CARDIA Study. AEP. 2010: 323-331.

Griep RH, Rotenberg L, Landsbergis P, Vasconcellos-Silva PR. Uso combinado de modelos de estresse no trabalho e a saúde auto-referida na Enfermagem. Rev Saude Publica. 2011; 45: 145-152. 
Grover SA, Coupal L, Hu XP. Identifying adults at increased risk of coronary disease. How well do the current cholesterol guidelines work? JAMA. 1995; 274(10), 801-806.

Hippisley-Cox J, Coupland C, Vinogradova Y, et al. Predicting cardiovascular risk in England and Wales: prospective derivation and validation of QRISK2. BMJ. 2008; 336(7659): 1475-1482.

Hintsa T, Kivimaki M, Elovainio M, Vahtera J, Hintsanen M, Viikari JS, et al. Is the association between job strain and carotid intima-media thickness attributable to preemployment environmental and dispositional factors? The ardioCvascular Risk in Young Finns Study. Occup Environ Med. 2008; 65: 676-682.

Hintsanen M, Elovainio M, Puttonen S, Kivimaki M, Lehtimaki T, Kahonen M, et al. $\mathrm{Val} / \mathrm{Met}$ polymorphism of the COMT gene moderates the association between job strain and early atherosclerosis in young men. J Occup Environ Med. 2008; 50: 649-657.

Jedryka-Goral A, Pasierski T, Zabek J, Widerszal-Bazyl M, Radkiewicz P, Szulczyk GA, et al. Risk factors for atherosclerosis in healthy employees-a multidisciplinary approach. Eur J Intern Med. 2006; 1: 247-253.

Johnson JV, Hall EM. Job Strain, Workplace Social Support and Cardiovascular Disease: a cross-sectional study of a random sample of the Swedish working population. Am J Public Health. 1988; 78:1336-1342. 
Kamarck TW, Muldoon MF, Shiffman S, Sutton-Tyrrell K, Gwaltney C, Janicki DL. Experiences of demand and control in a daily life as correlates of subclinical carotid atherosclerosis in a healthy older sample. Health Psychology. 2004: 23(1): 24-32.

Kamarck TW, Shiffman S, Sutton-Tyrrell K, Muldoon MF, Tepper P. Daily psychological demands are associated with 6-year progression of carotid artery atherosclerosis: the Pittsburgh Healthy Heart Project. Psychosom Med. 2012; 74: 432439.

Kamarck TW, Muldoon MF, Shiffman SS, Sutton-Tyrrell K. Experiences of demand and control during daily life are predictors of carotid atherosclerotic progression among healthy men. Health Psychol. 2007 May;26(3):324-32.

Karasek R, Brisson C, Kawakami N, Houtman I, Bongers P, Amick B. The Job Content Questionnaire (JCQ): an instrument for internationally comparative assessments of psychosocial job characteristics. J Occup Health Psychol. 1998; 3: 322-355.

Karasek R. Low social control and physiological deregulation - the stress disequilibrium theory, towards a new demand- control model. SJWEH. 2008;(6):117-135.

Kasliwal RR, Bansal M, Desai D, et al. Carotid intima-media thickness: Current evidence, practices, and Indian experience. Indian J Endocrinol Metab. 2014;18: 13-22.

Kasthiresan S, Larson MG, Keyes MJ, Polak JF, Wolf PA, D'Agostino RB, Jaffer FA, Clouse ME, Levy D, Manning WJ, O'Donnell CJ. 
Assessment by cardiovascular magnetic resonance, electron beam computed tomography, and carotid ultrasonography of the distribution of subclinical atherosclerosis across Framingham risk strata. Am J Cardiol. 2007 Feb $1 ; 99(3): 310-314$

Khot, UN, et al. Prevalence of conventional risk factors in patients with coronary heart disease. JAMA. 2003; 290(7),898-904.

Kuper H, Marmot M. Job strain, job demands, decision latitude, and risk of coronary heart disease within the Whitehall II Study. J Epidemiol Community Health. 2003; 57 (2): 14753.

Lauer MS. Primary prevention of atherosclerotic cardiovascular disease: the high public burden of low individual risk. JAMA. 2007; 297:1 376-1378.

Li Z, Froehlich J, Galis ZS, Fakatta EG. Increased expression of matrix metalloprotenase2 in the thickened intima of aged rats. Hypertension 1999; 33:116-23.

Mansur AP, Favarato D, Souza MF, Avakian SD, Aldrighi JM, et al. Trends in death from circulatory diseases in Brazil between 1979 and 1996. Arq Bras Cardiol. 2001; 76: 497510.

Mill JG, Pinto K, Griep RH, et al. Medical assessments and measurements in ELSABrasil. Rev Saude Publica. 2013; 47 (2): 54-62. 
Mookadam F, Moustafa SE, Lester SJ, et al. Subclinical Atherosclerosis: Evolving Role of Carotid Intima-Media Thickness. Prev Cardiol. 2010; 13: 186-197.

Muntaner C, Nieto FJ, Cooper L, Meyer J, Szklo M, Tyroler HA. Work organization and atherosclerosis: findings of the ARIC study. Am J Prev Med. 1998; 14(1): 9-18.

Nordstrom CK, Dwyer KM, Merz CN, Shircore A, Dwyer JH. Work-related stress and early atherosclerosis. Epidemiology. 2001;12: 180-185.

Paschoal T, Tamayo A. Validação da Escala de Estresse no Trabalho. Estudos de Psicologia. 2004; 9: 45-52.

Pereira AC, Gomez LM, Bittencourt MS, et al. Age, Gender, and Race-Based Coronary Artery Calcium Score Percentiles in the Brazilian Longitudinal Study of Adult Health (ELSA-Brasil). Clin Cardiol. 2016 Jun;39(6):352-9.

Ridker PM, Paynter NP, Rifai N et al. C-reactive protein and parental history improve global cardiovascular risk prediction: the Reynolds risk score for men. Circulation. 2008; 118: 2243-2251.

Rosenström T, Hintsanen M, Kivimäki M, Jokela M, Juonala M, Viikari JS, Raitakari OT, Keltikangas-Järvinen L. Change in job strain and progression of atherosclerosis: The Cardiovascular Risk in Young Finns study. J Occup Health Psychol. 2011; 16: 139-50. 
Rosvall M, Janzon L, Berglund G, Engstrom G, Hedblad B. Incident coronary events and case fatality in relation to common carotid intima-media thickness. $J$ Intern Med. 2005; 257:430-437.

Rosvall M, Östergren P, Hedblad B, Isacsson S, Janzon L, Berglund G. Socioeconomic differences in the progression of carotid atherosclerosis in middle-aged men and women with subclinical atherosclerosis in Sweden. Social Science and Medicine. 2006, 17851798.

Rosvall M, Östergren PO, Hedblad B, Isacsson SO, Janzon L, Berglund G. Work-related psychosocial factors and carotid atherosclerosis. Int J Epidemiol. 2002; 31: 1169-1178.

Ruijter HMD, Peters SAE, Anderson TJ, Briton AR et al. Common carotid intima-media thickness measurements in cardiovascular risk prediction: A Meta-analysis. JAMA. 2012; 308(8):796-803.

Rumberger JA, Simons DB, Fitzpatrick LA, et al. Coronary artery calcium area by electron-beam computed tomography and coronary atherosclerotic plaque area: a histopathologic correlative study. Circulation 1995; 92: 2157-2162.

Rundek T, Blanton SH, Bartels S, et al. Traditional risk factors are not major contributors to the variance in carotid intima-media thickness. Stroke 2013; 44: 2101-2108. 
Santos IS, Alencar AP, Rundek T, et al. Low Impact of Traditional Risk Factors on IMT:

The ELSA-Brasil Cohort. Arterioscler Thromb Vasc Biol. 2015; 35: 2054-59.

Santos IS, Bittencourt MS, Oliveira IR, et al. Carotid intima-media thickness value distributions in the Brazilian Longitudinal Study of Adult Health (ELSA-Brasil). Atherosclerosis. 2014; 237: 227-35.

Santos IS, Griep RH, Alves MG, et al. Job stress is associated with migraine in current workers: the Brazilian Longitudinal Study of Adult Health (ELSA-Brasil). Eur J Pain. 2014; 18: 1290-1297.

Schmidt MI, Duncan BB, Mill JG, Lotufo PA, Chor D, Barreto SM, Aquino EML, Passos VMA, Matos SMA, Molina MCB, Carvalho MS, Benseñor IM. Cohort Profile: Longitudinal Study of Adult Health (ELSA-Brasil). Int J Epidemiol. 2015 Feb;44(1):6875.

Sibley C, et al. Limitantions of current cardiovascular disease risk assessment strategies in women. Journal of Women's Health. 2002; 15(1),54-56.

Siegrist J. Adverse Health Effects of High-Effort / Low-Reward Conditions. J Occupational Health Psychology. 1996; 1: 27-41.

Simon A, Chironi G, Levenson J. Performance of subclinical arterial disease detection as a screening test for coronary heart disease. Hypertension. 2006; 48:392-396. 
Stein JH, Korcarz CE, Hurst RT, et al. Use of carotid ultrasound to identify subclinical vascular disease and evaluate cardiovascular disease risk: A consensus statement from the American Society of Echocardiography Carotid Intima-Media Thickness Task Force. Endorsed by the Society for Vascular Medicine. J Am Soc Echocardiogr 2008; 21: 93 111.

The IPAQ Research Committee. Guidelines for Data Processing and Analysis of the International Physical Activity Questionnaire (IPAQ) - Short Form and Long Forms. Available at: https://docs.google.com/viewer?a=v\&pid=sites\&srcid=ZGVmYXVsdGRvbWFpbnx0a GVpcGFxfGd4OjE0NDgxMDk3NDU1YWRIZTM. Published 2005. Accessed May 25, 2017.

Theorell T. Working conditions and health. In: Berkman L, Kawachi I, editors. Social epidemiology. New York: Oxford University Press; 2000. p. 95-118.

Theorell T, Karasek RA. Current Issues Relating to Psychosocial Job Strain and Cardiovascular Disease Research. J Occup Health Psychol. 1996; 1:9-26.

Toth PP. Subclinical atherosclerosis: what it is, what it means and what we can do about it? Int J Clin Pract. 2008, 62(8): 1246-1254. 
Touboul PJ, Hennerici MG, Meairs S, et al. Mannheim carotid intima-media thickness and plaque consensus (2004-2006-2011). An update on behalf of the advisory board of the $3 \mathrm{rd}$, 4th and 5th watching the risk symposia, at the 13th, 15th and 20th European Stroke Conferences, Mannheim, Germany, 2004, Brussels, Belgium, 2006, and Hamburg, Germany, 2011. Cerebrovasc Dis. 2012;34(4):290-6.

Wilson MD, Conroy LM, Dorevitch S. Occupational stress and subclinical atherosclerosis: a systematic review. Int J Occup Environm Health. 2014; 20: 271-280.

Xu W, Hang J, Cao T, Shi R, Zeng W, Deng Y, et al. Job stress and carotid intima-media thickness in Chinese workers. J Occup Health. 2010; 52: 257-262. 


\title{
8. Anexos:
}

Tabela 1 - Suplemento. O Questionário Demanda-Controle-Suporte Social.

\author{
Demandas Psicológicas
}

1. Com que frequência você tem que fazer suas tarefas de trabalho com muita rapidez?

2. Com que frequência você tem que trabalhar intensamente (isto é, produzir muito em pouco tempo)?

3. Seu trabalho exige demais de você?

4. Você tem tempo suficiente para cumprir todas as tarefas de seu trabalho?

5. O seu trabalho costuma apresentar exigências contraditórias ou discordantes?

Uso de habilidades

6. Você tem possibilidade de aprender coisas novas em seu trabalho?

7. Seu trabalho exige muita habilidade ou conhecimentos especializados?

8. Seu trabalho exige que você tome iniciativa?

9. No seu trabalho, você tem que repetir muitas vezes as mesmas tarefas?

Autoridade decisória

10. Você pode escolher COMO fazer o seu trabalho?

11. Você pode escolher O QUE fazer no seu trabalho? 
Suporte social no trabalho

12. Existe um ambiente calmo e agradável onde trabalho.

13. No trabalho, nos relacionamos bem uns com os outros.

14. Eu posso contar com o apoio dos meus colegas de trabalho.

15. Se eu não estiver num bom dia, meus colegas compreendem.

16. No trabalho, eu me relaciono bem com meus chefes.

17. Eu gosto de trabalhar com meus colegas. 


\section{User Menu}

Home

(/user/myprofile)

Manuscript

Manage E-Mail

Alerts

(/user/subscriptions)

Manage E-Mail

Newsletters

(/user/newsletter/list/subjects)

Manage Saved

Journal

Searches

(/user/mysearches)

Abstract

Change

Password

(/user/chgpwd)

Manage

Accounts

(/user/manage_accounts)

Edit Profile

(/user/edit)

Logout

(/user/logout)

Submissions
Menu

Submit

Manuscript

(/user/manuscripts/upload)

Display

Submitted

Manuscripts

(/user/manuscripts/status)

English Editing

(/user/pre_english_article/status)

Discount

Keywords

Vouchers

(/user/discount_voucher)

Invoices

Manuscript

(/user/invoices)

File

LaTex Word

Count

PDF File

(/user/get/latex_word_count) medicina-653276

Pending review

Article

Job stress and subclinical atherosclerosis: cross-sectional results of the Brazilian Longitudinal Study of Adult Health (ELSA-Brasil)

Medicina (https://www.mdpi.com/journal/medicina)

Background: Few studies evaluated the relationship of job stress with subclinical atherosclerosis. We aimed to evaluate the association of job stress and subclinical atherosclerosis in ELSA-Brasil. Methods: We considered job stress domains (demand, skill discretion, decision authority, and social support as independent variables and coronary artery calcium $(C A C>0 ; \geq 100)$ and carotid intima-media thickness (CIMT) $>$ P75 or as a continuous variable) as the dependent ones. Lower tertile was the reference for demand and higher tertile was the reference for the other dimensions. Odds ratio (OR) and $95 \%$ Confidence Interval $(95 \% \mathrm{Cl})$ were presented crude, adjusted for sociodemographic, cardiovascular risk factors and lifestyle variables. Linear regression models were built for CIMT as a continuous variable using the same adjustments. Results: After multivariate adjustment, the OR of CAC $>0$ for high demand was 1.15 (0.89-1.49); for low skill discretion 0.94 $(95 \% \mathrm{Cl}, 0.74-1.21)$; for decision authority $0.88(95 \% \mathrm{Cl}, 0.71-$ 1.098); and for low social support 1.20 (95\%Cl, 0.96-1.49). Similar results were obtained for $C A C \geq 100$. For CIMT, after multivariate adjustment, the OR for high demand was 1.07 $(95 \% \mathrm{Cl}, 0.84-1.36)$; for low skill discretion $1.07(95 \% \mathrm{Cl}, 0.84-$ 1.35); for low decision authority $1.13(95 \% \mathrm{Cl}, 0.84-1.27)$; and for low social support $0.92(95 \% \mathrm{Cl}, 0.75-1.14)$. Using linear regression models, there was an association of low and middle decision authority and social support with lower CIMT levels, but all results lost significance after further adjustment for sociodemographic variables. Discussion: Our results did not support an association of job stress with subclinical atherosclerosis measured by CAC or CIMT.

job strain, subclinical atherosclerosis, coronary artery calcification, carotid intima-media thickness, cardiovascular disease.

manuscript.docx

(/user/manuscripts/displayFile/4740b9fff001096256eda0b9c6 0e3450)

manuscript.pdf

(/user/manuscripts/displayFile/4740b9fff001096256eda0b9c6 0e3450/latest_pdf)

Help

(/user/manuscripts/he Preprints

You can put your paper online immediately and before peer review at Preprints.org (https://www.preprints.org), with the

$\checkmark$ Reviewers following benefits: 


\section{Volunteer}

Preferences

(/volunteer_reviewer_info/view)

- Anyone can read and download your work immediately, before peer review is complete.

- Receive comments and feedback.

- Make your work citable via assignment of a digital object identifier.

- Immediate indexing by Google Scholar and other online databases.

- Papers are put online within 24 hours.

Upload to Preprints (/user/sciprints/manuscript/4740b9fff001096256eda0b9c60e 3450)

Data is of paramount importance to scientific progress, yet most research data drowns in supplementary files or remains private. Enhancing the transparency of the data processes will help to render scientific research results reproducible and thus more accountable. Co-submit your methodical data processing articles or data descriptors for a linked data set in Data (https://www.mdpi.com/journal/data) journal to make your data more citable and reliable.

- Deposit your data set in an online repository, obtain the DOI number or link to the deposited data set.

- Download and use the Microsoft Word template (https://www.mdpi.com/files/word-templates/datatemplate.dot) or LaTeX template (https://www.mdpi.com/authors/latex) to prepare your data article.

- Upload and send your data article to the Data (https://www.mdpi.com/journal/data) journal here (/user/manuscripts/upload? form\%5Bjournal_id\%5D=176\&form\%5Barticle_type_id\%5D=47).

Submit To Data (/user/manuscripts/upload? form\%5Bjournal_id $\% 5 \mathrm{D}=176 \&$ form $\% 5$ Barticle_type_id $\% 5 \mathrm{D}=$ 47)

\section{Author Information}

Submitting Luciana Bivanco de L. Lorentz

Author

Corresponding Isabela Judith M. Benseñor Author

Author \#1 Luciana Bivanco de L. Lorentz

E-Mail lulisbivanco@yahoo.com.br

Author \#2 Itamar de Souza Santos

E-Mail_itamarss@usp.br

Author \#3 Márcio Sommer Bittencourt

E-Mail marciosbittencourt@gmail.com 
Author \#4 Rosane H. Griep

E-Mail rohgriep@ioc.fiocruz.br

Author \#5 Paulo Andrade Lotufo

E-Mail_palotufo@usp.br

Author \#6 Isabela Judith M. Benseñor

E-Mail isabensenor@gmail.com

Manuscript Information

$\begin{aligned} \begin{array}{r}\text { Received } \\ \text { Date }\end{array} & 12 \text { November } 2019 \\ \text { Page Count } & 23\end{aligned}$

APC information

Journal $\quad 1,500.00 \mathrm{CHF}$

APC:

Total $\quad 1,500.00 \mathrm{CHF}$

Payment

Amount:

\section{Related Papers Published in MDPI Journals}

If you have any questions or concerns, please do not hesitate to contact medicina@mdpi.com (mailto: medicina@mdpi.com).

(C) 1996-2019 MDPI (Basel, Switzerland) unless otherwise stated 
São Paulo, 25 de setembro de 2017.

$I^{m o(a)} \cdot S^{r(a)}$

Profa. Dra. Isabela Judith Martins Benseñor

Centro de Pesquisa Clinica e Epidemiológica

UNIVERSIDADE DE SÃO PAULO

REFERENTE: Projeto de Pesquisa "O papel do estresse laboral nos marcadores de Aterosclerose Subclínica"

Pesquisadora responsável: Profa. Dra. Isabela Judith Martins Benseñor

Pesquisadora responsável: Luciana Bivanco de Lima

CAAE: 74289317.0 .0000 .0076

Registro CEP-HU/USP: $1667 / 17$

Prezado(a) Senhor(a)

O Comitê de Ética em Pesquisa do Hospital Universitário da Universidade de São Paulo, em reunião ordinária realizada no dia 15 de setembro de 2017 analisou o Projeto de Pesquisa acima citado, considerando-o como APROVADO.

Lembramos que cabe ao pesquisador elaborar e apresentar a este Comitê, relatórios parciais semestrais e final, de acordo com a Resolução n ${ }^{\circ} 466 / 2012$ do Conselho Nacional de Saúde, inciso XI.2, letra "d".

O primeiro relatório está previsto para 15 de março de 2018.

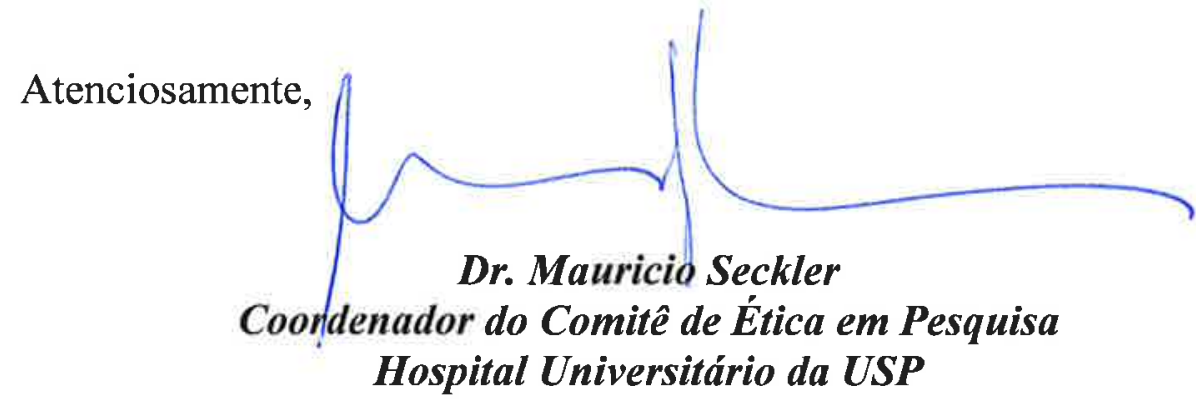

